\title{
Numerical assessment of the scattering of acoustic waves by turbulent structures
}

\author{
V. Clair* and G. Gabard ${ }^{\dagger}$ \\ Institute of Sound and Vibration Research, University of Southampton, UK.
}

\begin{abstract}
The scattering of harmonic sound waves by a single vortex is studied to provide some insight into the more complex problem of sound scattering by a turbulent layer. Two different methods are used to model the scattering by a steady vortex or by a vortex convected in a uniform mean flow. The first method is based on the linearized Euler equations in the frequency domain and is used to study the scattering by a steady vortex. The speed of this method allows to perform a study of the spatial scattering of a plane wave over a wide range of frequencies, where analytical models previously developed are restricted either to low or high frequencies. The second method uses a finite difference solver, also based on the linearized Euler equations but in the time domain. This method is used to study the scattering by a vortex convected in a uniform mean fow, where a spectral scattering occurs in addition to the spatial scattering. A parametric study is realized, focusing on four parameters including the source frequency and the convection velocity. The spectra deduced from this study show a pattern similar to the haystacking observed in existing studies of the scattering by a turbulent shear layer. Some of the trends deduced from the parametric study are also in agreement with these previous observations. Thus, the detailled study of the scattering by a single eddy is able to provide further understanding of the turbulence scattering.
\end{abstract}

\section{Introduction}

When acoustic waves propagate through a volume of turbulence, a scattering phenomenon occurs leading to a spatial and spectral redistribution of the acoustic energy. This scattering is observed, for example, when the tones generated by the turbine stage of a turbofan engine are radiated from the exhaust nozzle. These tones then propagate through the turbulent shear layers formed between the jet and the bypass stream, and also between the bypass stream and the free stream. The scattering by these shear layers leads to a reduction of the power of the tones to the benefit of a broadband field around the tones frequencies. Similarly, when experiments are performed in an open jet wind tunnel with microphones located outside of the jet core, the scattering by the turbulent shear layer of the jet can alter the amplitude of the tonal components measured. Turbulent scattering also received some attention in the domain of atmospheric propagation, when acoustic sounding techniques were studied as a mean to deduce characteristics of a turbulent atmosphere (such as the mean wind profile or spatial spectra of the velocity field).

Turbulent scattering has been studied originally by Lighthill ${ }^{21}$ and Kraichnan, ${ }^{19}$ using Lighthill's acoustic analogy, assuming that the scattering is weak (meaning that the proportion of scattered energy is small relative to the energy of the incident field). Lighthill's analysis was focused on the spatial scattering of energy while Kraichnan also derived expressions for the spectrum, especially when considering an isotropic turbulence. Howe ${ }^{18}$ developed kinetic equations in which the different terms of the equations can be linked either to the spatial or spectral redistribution of energy. This model is able to consider multiple scattering, whereas previous models were restricted to the hypothesis of single scattering. Turbulent scattering was also studied by Brown ${ }^{2}$ and Brown \& Clifford ${ }^{3,4}$ in the context of propagation through a turbulent atmosphere.

\footnotetext{
${ }^{*}$ Research Fellow, Institute of Sound and Vibration Research, University of Southampton. SO17 1BJ Southampton, UK. V.J.Clair@soton.ac.uk

${ }^{\dagger}$ Associate Professor, Institute of Sound and Vibration Research, University of Southampton. SO17 1BJ Southampton, UK. AIAA Member.
} 
They developed a model based on the resolution of an inhomogeneous Helmholtz equation with a source term including velocity and temperature fluctuations. In the same context, Goedecke et al. ${ }^{17}$ developed a semianalytical model based on the scattering by a single eddy, later coupled to a numerical computation in which a turbulent volume is successively split into cells of different sizes, each containing an eddy. The scattering of an acoustic radiation by a turbulent shear layer has been investigated experimentally by Candel et al..$^{7,8}$ considering an harmonic source on the jet centerline. The results showed a specific shape of the acoustic spectra consisting in an attenuated peak at the source frequency surrounded by two sidebands (or haystacks). In this experimental study and in the recent measurements for the project GARTEUR AD/AG-50, ${ }^{20,26}$ the evolutions of the haystacks shape with several parameters of the jet flow and the incident frequency were investigated. These configurations have also been studied analytically by Campos ${ }^{5,6}$ who concluded that for an engine jet, widening the shear layer could significantly reduce tonal components propagating through the exhaust. Powles et al..$^{25}$ and McAlpine et al. ${ }^{22}$ developed an analytical model to predict the haystacking of turbine tones. This model is able to consider frozen or evolving turbulence, and the variations of the source/flow parameters show behaviors of the spectra agreeing with existing experimental results. Ewert et al. ${ }^{12,13}$ perfomed first attempts of numerical calculations for such configurations solving the linearized Euler equations (LEE) in the time domain with a finite difference solver and using the stochastic RPM method in order to synthesize and introduce the turbulence in the numerical domain. The method showed promising results in its ability to recover features of the spectral broadening and its evolution with several parameters of the source or the flow.

Most of the studies on the scattering of acoustic waves by a turbulent layer concludes that the broadening of the spectrum is mainly due to the motion of the large turbulent structures, which are bearing most of the energy. In order to study more precisely the effects of the size of these structures and their motion, it is of interest to study the scattering of an acoustic wave by a single vortex, instead of a complete turbulent layer. The scattering of a monopole or a plane wave by a steady vortex has been studied analytically by O'Shea. ${ }^{24}$ The model is based on Lighthill's analogy and uses asymptotic expansions in order to derive expressions for the scattered field, in the Born limit (or low-frequency limit) and assuming that the flow field is small compared to the speed of sound. However, these expressions involve an infinite scattering in the forward direction due to a diverging integral. Colonius et al. ${ }^{9}$ tackled this issue by solving this integral exactly, using a Fourier expansion in the azimuthal direction. Ford \& Llewellyn Smith ${ }^{15}$ also developed an analytical model for this configuration, under the same assumptions regarding the frequency and the flow, but this model is based on asymptotic expansions of Euler's equations. Their predictions show scattering patterns very similar to the results of Colonius et al. ${ }^{9}$ model. For high frequencies, Georges ${ }^{16}$ suggested $^{2}$ the use of a ray tracing technique to determine the ray paths and Colonius et al. ${ }^{9}$ extended this approach by calculating the amplitudes and phases of the waves. In addition to their analytical modeling, Colonius et al. ${ }^{9}$ performed direct numerical simulations of the scattering of a plane wave by a steady vortex. These simulations confirmed that the analytical models are able to predict correct trends for the scattered field, but some differences appear because of higher order effects discarded in the analytical solutions.

In the present study, a semi-numerical method based on the resolution of the LEE in the frequency domain, decomposed over a sum of azimuthal modes, is proposed in order to study the scattering of a plane wave by a steady vortex. This method does not suffer from the restrictions associated with analytical models, and it is therefore possible to consider both low and high frequency incident waves. In addition, the low computational cost of the method allows to perform a study over a large number of frequencies and thus, to show the transition and the drastic changes in the scattering pattern between the low and high frequency domains. In all the models described above, the vortex is steady, therefore there is no relative motion between the source, the vortex, and the observers. As a result, there is no spectral redistribution of the energy. In the present paper, the scattering of acoustic waves by a vortex convected in a uniform mean flow is also investigated. The study relies on the use of a second numerical method consisting in solving the LEE in the time domain with a finite difference solver, considering an unsteady base flow instead of a classical steady mean flow. The effects of several parameters such as the position of the source, the frequency of the source, the convection velocity and the magnitude of the vortex are assessed through a parametric study. The results show that, even in this simple configuration, the spectra display sidebands around the peak frequency that are similar to the haystacks observed in studies of the scattering by a turbulent shear layer. Moreover, the evolution of these sidebands with the parameters considered in this work shows trends that were also found for a turbulent shear layer. In the present study, the apparition of the sidebands and their evolution are coherent with an explanation based on observations made on the spatial scattering by a 
vortex, associated with a Doppler effect due to the motion of the vortex relative to the acoustic source and the microphone.

The first part of the paper presents the configuration considered and the expressions of the vortex flow as well as a description of the two methods used in this study. In the second part, a validation case for a steady vortex based on Colonius et al. ${ }^{9}$ is performed with both of the methods, and the semi-numerical model is used to perform a study of the spatial scattering over a wide range of frequencies. Finally, the spectral broadening of an harmonic sound wave by a convected vortex is studied and the effects of varying the parameters mentioned before are assessed.

\section{Presentation of the problem studied and the numerical methods used}

\section{II.A. Description of the configuration studied}

The configuration studied here consists in an acoustic field (point source or plane wave) scattered by a single vortex, either steady or convected by a uniform mean flow, as illustrated in figure 1 . When the vortex is stationnary, we study the spatial distribution of the scattered field. When the vortex is convected, several microphones are positionned in the computational domain in order to assess the effect of the scattering on the sound pressure level (SPL) spectra.

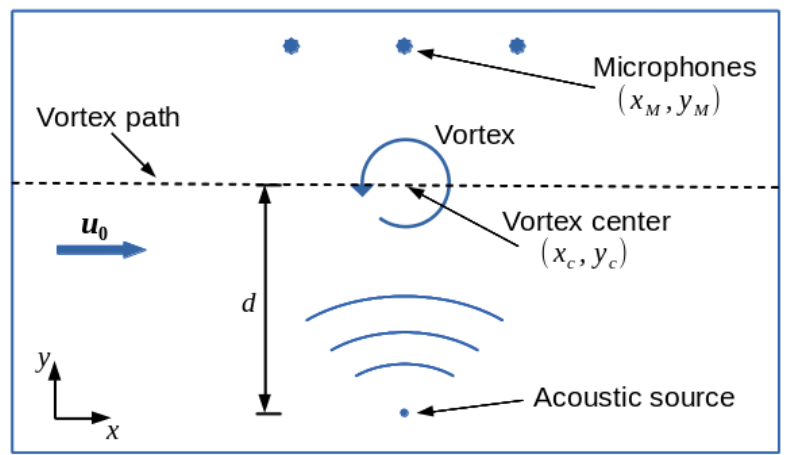

Figure 1: Schematic of the scattering of an acoustic radiation by a convected vortex.

In this paper, the vortex considered is inviscid and its flow field is similar to the zero-circulation vortex of Colonius et al. ${ }^{9}$ The expression of the velocity field of the vortex presented below derives from a Gaussian stream function, and the pressure and density field are defined so that the flow is solution of the Euler equations:

$$
\left\{\begin{array}{l}
u_{r}^{t}(r, \theta, t)=0 \\
u_{\theta}^{t}(r, \theta, t)=\frac{U_{v} r(t)}{L} \exp \left(\frac{1}{2}\left[1-\frac{r(t)^{2}}{L^{2}}\right]\right) \\
p^{t}(r, \theta, t)=\frac{\rho_{0} c_{0}^{2}}{\gamma}\left[1-\frac{(\gamma-1) U_{v}^{2}}{2 c_{0}^{2}} \exp \left(1-\frac{r(t)^{2}}{L^{2}}\right)\right]^{\frac{\gamma}{\gamma-1}} \\
\rho^{t}(r, \theta, t)=\left[\gamma p^{t}(r, \theta, t)\right]^{\frac{1}{\gamma}}
\end{array}\right.
$$

where $\rho_{0}$ and $c_{0}$ are the free field density and speed of sound, $\gamma$ is the heat capacity ratio, and $U_{v}$ is the velocity magnitude of the vortex (we also define the associated Mach number of the vortex $\mathrm{M}_{v}=U_{v} / c_{0}$ ). $L$ is the vortex core size, which corresponds to the radial position where the velocity of the vortex is maximum. An example of the radial evolution of the vortex velocity for a Mach number of the vortex $\mathrm{M}_{v}=0.05$ and a core size $L=10$ is shown in figure 2 . If the vortex is convected by a uniform mean flow $\boldsymbol{u}_{0}$, the position of its center $\boldsymbol{x}_{c}$ is evolving with time as $\boldsymbol{x}_{c}(t)=\boldsymbol{x}_{c}(0)+\boldsymbol{u}_{0} t$, and the radius is defined as $r(t)=\sqrt{\left(x-x_{c}(t)\right)^{2}+\left(y-y_{c}(t)\right)^{2}}$. 


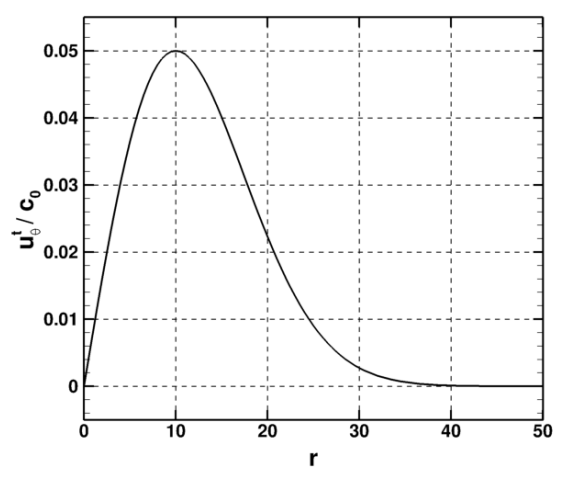

Figure 2: Radial evolution of the vortex velocity with $\mathrm{M}_{v}=0.05$ and $L=10$.

\section{II.B. LEE in the time domain for the scattering by a convected vortex}

The PIANO solver, developed by the DLR, is used in order to solve the linearized Euler equations (LEE) in the time domain. This solver has been used in order to study a vast range of problems in acoustics, notably problems involving interactions with turbulence. ${ }^{10,11,14}$

In order to capture the interaction between the incident acoustic field and the convected vortex, the equations solved have to contain terms involving products between the acoustic fluctuations and the vortex flow. A set of equations has been developed by Ewert et al. ${ }^{13}$ by considering small perturbations $\left(\rho^{\prime}, \boldsymbol{u}^{\prime}, p^{\prime}\right)^{T}$ over an unsteady base flow $(\rho, \boldsymbol{u}, p)^{T}$ instead of the usual steady mean flow $\left(\rho_{0}, \boldsymbol{u}_{0}, p_{0}\right)^{T}$. The linearized equations resolved become:

$$
\begin{aligned}
& \frac{\partial \rho^{\prime}}{\partial t}+\boldsymbol{u} \cdot \nabla \rho^{\prime}+\boldsymbol{u}^{\prime} \cdot \nabla \rho+\rho \nabla \cdot \boldsymbol{u}^{\prime}+\rho^{\prime} \nabla \cdot \boldsymbol{u}=0, \\
& \frac{\partial \boldsymbol{u}^{\prime}}{\partial t}+(\boldsymbol{u} \cdot \nabla) \boldsymbol{u}^{\prime}+\left(\boldsymbol{u}^{\prime} \cdot \nabla\right) \boldsymbol{u}+\frac{\nabla p^{\prime}}{\rho}-\frac{\nabla p \rho^{\prime}}{\rho^{2}}=\mathbf{0}, \\
& \frac{\partial p^{\prime}}{\partial t}+\boldsymbol{u} \cdot \nabla p^{\prime}+\boldsymbol{u}^{\prime} \cdot \nabla p+\gamma p \nabla \cdot \boldsymbol{u}^{\prime}+\gamma p^{\prime} \nabla \cdot \boldsymbol{u}=0 .
\end{aligned}
$$

The unsteady base flow is then decomposed in a steady mean part $\bullet_{0}$ and a fluctuating part ${ }^{t}$ (here the vortex flow defined in Eq. (1)): $(\rho, \boldsymbol{u}, p)^{T}=\left(\rho_{0}, \boldsymbol{u}_{0}, p_{0}\right)^{T}+\left(\rho^{t}, \boldsymbol{u}^{t}, p^{t}\right)^{T}$.

Some observations can be made on the interaction terms between the acoustic field and the unsteady flow that will appear because of this decomposition of the base flow. First, the unsteady part of the mean flow is assumed to be divergence-free $\left(\nabla \cdot \boldsymbol{u}^{t}=0\right)$, which means that it does not introduce additional sound sources. This hypothesis is true for the vortex flow defined in Eq.(1). The second assumption introduced by Ewert et al. ${ }^{13}$ is that the scattering of the incident acoustic field is mainly induced by the unsteady velocity fluctuations. Thus the effects of the unsteady density and pressure fluctuations are negligible. This assumption is assessed numerically in Sec. III for the case of a steady vortex. Thus, the interaction terms that are considered to be responsible of the acoustic scattering in Eq. (2) are the following:

$$
\begin{aligned}
h_{1} & =\boldsymbol{u}^{t} . \nabla \rho^{\prime}, \\
\boldsymbol{h}_{2} & =\left(\boldsymbol{u}^{t} . \nabla\right) \boldsymbol{u}^{\prime}+\left(\boldsymbol{u}^{\prime} . \nabla\right) \boldsymbol{u}^{\boldsymbol{t}}, \\
h_{3} & =\boldsymbol{u}^{t} . \nabla p^{\prime} .
\end{aligned}
$$

Ewert et al. ${ }^{12,13}$ suffered from growing numerical instabilities when trying to solve these equations, because of the terms of Eq.(2) involving mean flow gradients. In the present paper, the steady mean flow considered is uniform, therefore there is no gradients and the equations do not require any specific treatment.

The spatial discretization of this set of equations is realized in PIANO using the 7 points stencil, $4^{\text {th }}$ order DRP finite differences scheme by Tam \& Webb, ${ }^{27}$ and an explicit $8^{\text {th }}$ order filter is applied in order to prevent high frequency spurious oscillations to appear in the computational domain. The time integration is performed using a $4^{\text {th }}$ order Runge-Kutta time marching scheme with 4 stages. A coordinate transformation is applied in order to deal with curvilinear meshes encountered for complex geometries, and the solver is 
parallelized using the MPI library. In the computations realized in sections III and IV, the acoustic sources considered are either a plane wave or a monopole located relatively far from the vortex path. In order to reduce the computational effort, the monopole radiation is introduced into the numerical domain through a sponge layer located next to a boundary (the lower boundary in section IV).

\section{II.C. Solving the LEE in the frequency domain with a modal decomposition}

\section{II.C.1. Equations solved for a steady vortex}

This method has been developed in order to study the scattering of an harmonic plane wave by a steady vortex flow as presented in Eq. (1) with no convection flow. The Euler equations can therefore be linearized around this vortex flow, denoted $\boldsymbol{q}=(\rho, \boldsymbol{u}, p)^{T}$ in this section, in order to get a set of equations for the pressure, density and velocity fluctuations $\boldsymbol{q}^{\prime}=\left(\rho^{\prime}, \boldsymbol{u}^{\prime}, p^{\prime}\right)^{T}$ :

$$
\frac{\partial \boldsymbol{q}^{\prime}}{\partial t}+\boldsymbol{A} \boldsymbol{q}^{\prime}+\boldsymbol{B} \frac{\partial \boldsymbol{q}^{\prime}}{\partial r}+\boldsymbol{C} \frac{\partial \boldsymbol{q}^{\prime}}{\partial \theta}=\mathbf{0}
$$

with

$$
\begin{gathered}
\boldsymbol{A}=\left(\begin{array}{cccc}
0 & \frac{\rho}{r}+\frac{\partial \rho}{\partial r} & 0 & 0 \\
\frac{-u_{\theta}^{2}}{\rho r} & 0 & \frac{-2 u_{\theta}}{r} & 0 \\
0 & \frac{\partial u_{\theta}}{\partial r}+\frac{u_{\theta}}{r} & 0 & 0 \\
0 & \frac{\gamma p}{r}+\frac{\partial p}{\partial r} & 0 & 0
\end{array}\right) \quad, \quad \boldsymbol{B}=\left(\begin{array}{cccc}
0 & \rho & 0 & 0 \\
0 & 0 & 0 & \frac{1}{\rho} \\
0 & 0 & 0 & 0 \\
0 & \gamma p & 0 & 0
\end{array}\right) \\
\boldsymbol{C}=\left(\begin{array}{cccc}
\frac{u_{\theta}}{r} & 0 & \frac{\rho}{r} & 0 \\
0 & \frac{u_{\theta}}{r} & 0 & 0 \\
0 & 0 & \frac{u_{\theta}}{r} & \frac{1}{\rho r} \\
0 & 0 & \frac{\gamma p}{r} & \frac{u_{\theta}}{r}
\end{array}\right)
\end{gathered}
$$

The fluctuations $\boldsymbol{q}^{\prime}$ are written as the sum of the incident wave $\boldsymbol{q}_{i}^{\prime}=\left(\rho_{i}^{\prime}, \boldsymbol{u}_{i}^{\prime}, p_{i}^{\prime}\right)^{T}$ and a scattered wave $\boldsymbol{q}_{s}^{\prime}=\left(\rho_{s}^{\prime}, \boldsymbol{u}_{s}^{\prime}, p_{s}^{\prime}\right)^{T}: \boldsymbol{q}^{\prime}=\boldsymbol{q}_{i}^{\prime}+\boldsymbol{q}_{s}^{\prime}$. If the incident wave considered is a plane wave in the $x$-direction, the pressure can be written in cylindrical coordinates:

$$
p_{i}^{\prime}(r, \theta, t)=\mathrm{e}^{\mathrm{i}\left(\omega_{0} t-k_{0} r \cos \theta\right)}
$$

where $k_{0}=\omega_{0} / c_{0}$ is the free-field wavenumber. By applying a modal decomposition, the incident pressure field and the associated density and velocity fields become:

$$
\boldsymbol{q}_{i}^{\prime}(r, \theta, t)=\sum_{m}\left(\begin{array}{c}
\frac{(-\mathrm{i})^{m}}{c_{0}^{2}} \mathrm{~J}_{m}\left(k_{0} r\right) \\
\frac{(-\mathrm{i})^{m-1}}{\rho_{0} c_{0}} \mathrm{~J}_{m}^{\prime}\left(k_{0} r\right) \\
\frac{m(-\mathrm{i})^{m}}{\rho_{0} \omega_{0} r} \mathrm{~J}_{m}\left(k_{0} r\right) \\
(-\mathrm{i})^{m} \mathrm{~J}_{m}\left(k_{0} r\right)
\end{array}\right) \mathrm{e}^{\mathrm{i}\left(\omega_{0} t-m \theta\right)}
$$

where $\mathrm{J}_{m}$ is the $\mathrm{m}^{\text {th }}$ order Bessel function of the first kind.

The scattered field is also expressed in terms of a sum of azimuthal modes with an angular frequency $\omega_{0}$ :

$$
\boldsymbol{q}_{s}^{\prime}(r, \theta, t)=\sum_{m} \boldsymbol{q}_{s, m}^{\prime}(r, \theta, t) \mathrm{e}^{\mathrm{i}\left(\omega_{0} t-m \theta\right)} \quad, \text { thus } \quad \boldsymbol{q}^{\prime}(r, \theta, t)=\sum_{m} \boldsymbol{q}_{m}^{\prime}(r, \theta, t) \mathrm{e}^{\mathrm{i}\left(\omega_{0} t-m \theta\right)}
$$

The linearized Euler equations can then be written for each azimuthal order $m$ as the following system:

$$
\mathrm{i} \omega_{0} \boldsymbol{q}_{m}^{\prime}+\boldsymbol{A} \boldsymbol{q}_{m}^{\prime}+\boldsymbol{B} \frac{\partial \boldsymbol{q}_{m}^{\prime}}{\partial r}-\mathrm{i} m \boldsymbol{C} \boldsymbol{q}_{m}^{\prime}=\mathbf{0}
$$

After discretization of the radial derivatives of $\boldsymbol{q}_{m}^{\prime}$ by the spatial finite difference scheme (here a 7 points, 4th order DRP scheme) over an array $\breve{\boldsymbol{r}}$ representing the radial direction, the system can be written under the form: 


$$
\boldsymbol{X} \breve{\boldsymbol{q}}_{\boldsymbol{m}}^{\prime}+m \boldsymbol{Y} \breve{\boldsymbol{q}}_{\boldsymbol{m}}^{\prime}=0
$$

The solution matrix containing the variables at every node is splitted as $\breve{\boldsymbol{q}}_{\boldsymbol{m}}^{\prime}=\breve{\boldsymbol{q}}_{\boldsymbol{i}, \boldsymbol{m}}^{\prime}+\breve{\boldsymbol{q}}_{\boldsymbol{s}, \boldsymbol{m}}^{\prime}$, and the system on the scattered field becomes:

$$
\boldsymbol{X} \breve{\boldsymbol{q}}_{\boldsymbol{s}, \boldsymbol{m}}^{\prime}+m \boldsymbol{Y} \breve{\boldsymbol{q}}_{\boldsymbol{s}, \boldsymbol{m}}^{\prime}=\boldsymbol{g}_{i, m} \quad, \quad \boldsymbol{g}_{i, m}=-\boldsymbol{X} \breve{\boldsymbol{q}}_{\boldsymbol{i}, \boldsymbol{m}}^{\prime}-m \boldsymbol{Y} \breve{\boldsymbol{q}}_{\boldsymbol{i}, \boldsymbol{m}}^{\prime}
$$

In order to use the centered DRP scheme for the first and last points of the radial array, three additional points are created on each side of the array $\breve{\boldsymbol{r}}$. The points created on the $\breve{r}=0$ side have negative radii values. Based on Mohseni and Colonius, ${ }^{23}$ the values imposed at these points for scalar fields (density, pressure) and vector fields (velocity) are:

$$
\left(\begin{array}{c}
\rho^{\prime}(r) \\
\boldsymbol{u}^{\prime}(r) \\
p^{\prime}(r)
\end{array}\right)=\left(\begin{array}{c}
\rho^{\prime}(-r) \\
-\boldsymbol{u}^{\prime}(-r) \\
p^{\prime}(-r)
\end{array}\right) \mathrm{e}^{-\mathrm{i} m \pi}
$$

For the points created on the $\breve{r}=\breve{r}_{\max }$ side, the values of the variables are written as functions of the last real point $\left(\breve{r}=\breve{r}_{\max }\right)$ using a procedure similar to the far-field expansion described below in Eq.(12). In the far-field (far from any flow perturbation due to the vortex), the scattered pressure can be expressed using a multipole expansion with modes amplitudes $A_{m}$ deduced from the pressure field calculated over the array $\breve{\boldsymbol{r}}$. The velocity field is deduced from the linearized Euler equations in Eq.(8) with a zero mean velocity: $\mathrm{i} \omega_{0} \rho_{0} \boldsymbol{u}_{\boldsymbol{s}}+\nabla p_{s}=0$ :

$$
\boldsymbol{q}_{s}^{\prime}(r, \theta, t)=\sum_{m} A_{m}\left(\begin{array}{c}
\frac{1}{c_{\infty}^{2}} \mathrm{H}_{m}^{(2)}\left(k_{0} r\right) \\
\frac{\mathrm{i}}{\rho_{0} c_{\infty}} \mathrm{H}_{m}^{(2)^{\prime}}\left(k_{0} r\right) \\
\frac{m}{\rho_{0} \omega_{0} r} \mathrm{H}_{m}^{(2)}\left(k_{0} r\right) \\
\mathrm{H}_{m}^{(2)}\left(k_{0} r\right)
\end{array}\right) \mathrm{e}^{\mathrm{i}\left(\omega_{0} t-m \theta\right)}
$$

where $\mathrm{H}_{m}^{(2)}$ is the $\mathrm{m}^{\text {th }}$ order Hankel function of the second kind.

If the observer is chosen very far from the vortex, an asymptotic expression of the Hankel function can be used and the pressure expression in Eq.(12) becomes:

$$
p_{s}^{\prime}(r, \theta, t)=\frac{D(\theta)}{\sqrt{k_{0} r}} \mathrm{e}^{\mathrm{i}\left(\omega_{0}\left[t-r / c_{\infty}\right]\right)}
$$

with

$$
D(\theta)=\sqrt{\frac{2 \mathrm{i}}{\pi}} \sum_{m} \mathrm{i}^{m} A_{m} \mathrm{e}^{-\mathrm{i} m \theta}
$$

\section{II.C.2. Doppler corrections for the estimation of the spectral broadening}

If the vortex is convected by a uniform mean flow, there are two corrections that have to be accounted for in order to reproduce the spectral broadening observed at a fixed microphone position. First, due to the convection of the vortex by the mean flow, the effective frequency seen by the vortex is modified. This frequency shift depends on the angle $\alpha$ between the mean flow direction and the plane wave propagation direction. The frequency seen by the vortex $\tilde{\omega}_{0}$ is:

$$
\tilde{\omega}_{0}=\frac{\omega_{0}}{1+M \cos (\alpha)}
$$

The second correction comes from the relative movement between the vortex and the microphone. If we suppose a uniform mean flow with velocity $u_{0}$ in the $x$-direction, the position of a microphone located in $\left(x_{M}, y_{M}\right)$ in the fixed frame will evolve with time in the reference frame attached to the vortex:

$$
\left\{\begin{array}{l}
\tilde{x}_{M}(t)=x_{M}-u_{0} t \\
\tilde{y}_{M}(t)=y_{M}
\end{array}\right.
$$


or in polar coordinates:

$$
\left\{\begin{array}{l}
\tilde{r}_{M}(t)=\sqrt{\tilde{x}_{M}^{2}(t)+\tilde{y}_{M}^{2}(t)} \\
\tilde{\theta}_{M}(t)=\tan ^{-1}\left(\frac{\tilde{y}_{M}(t)}{\tilde{x}_{M}(t)}\right)
\end{array}\right.
$$

The time-evolving scattered pressure at the microphone position $p_{s, M}^{\prime}$ can then be expressed using eq. (13) (we suppose that the microphone is far enough from the vortex to use this asymptotic expression) and taking the Doppler corrections into account:

$$
p_{s, M}^{\prime}(t)=\frac{D(\tilde{\theta}(t)-\alpha)}{\sqrt{\tilde{k_{0}} \tilde{r}(t)}} \mathrm{e}^{\mathrm{i}\left(\tilde{\omega}_{0}\left[t-\tilde{r}(t) / c_{\infty}\right]\right)}
$$

Note that the directivity of the scattered field $D(\theta)$ has been rotated by the angle of incidence of the plane wave $\alpha$.

\section{Scattering of a plane wave by a steady vortex}

\section{III.A. Validation on a reference case by Colonius et al.}

This test case consists in calculating the scattering of a plane wave (with $\lambda_{0}=4 L$ ) propagating in the $+x$-direction by a stationary vortex having a maximum velocity $\mathrm{M}_{v}=0.125$. In PIANO, the vortex core $L$ is discretized by 8 points with a regular cell size $\Delta x=\Delta y=1$. The mesh extends between $-10 L \leq x \leq 30 L$ and $-20 L \leq y \leq 20 L$, and two sponge layers are added upstream (50 points for the injection of the plane wave) and downstream (100 points to dissipate the acoutic fluctuations before they reach the boundary) leading to a $471 \times 321$ points mesh. A Thompson's ${ }^{28}$ 1-D characteristic boundary condition is applied at each boundary. The time step is chosen to ensure the CFL condition everywhere in the domain. The calculation is runned with and without the vortex until a periodic state is reached for the acoustic field. Then, the acoustic fields with and without the vortex are recorded at each iteration during a period of the source in order to compute the scattered field (by substracting the acoustic field without the vortex to the acoutic field with the vortex) and its RMS value.

A snapshot of the total acoustic pressure (reduced by the amplitude of the incident wave $P_{i}$ ) is presented in figure 3 , showing alterations of the wavefronts downstream of the vortex. The dissipation by the sponge layer is also observable next to the outflow boundary. The scattered pressure field is plotted in figure 4(a). It displays two main scattering directions which seems relatively symmetrical relative to the $y=0$ axis. The RMS field of the scattered pressure presented in figure 4(b) shows again the two main scattering directions, but a slight assymetry relative to $y=0$ is visible. The effects of some spurious reflexions near the top and bottom boundaries can be observed, because the boundary conditions applied are only designed to let waves with a propagation direction normal to the boundary leave the domain properly.

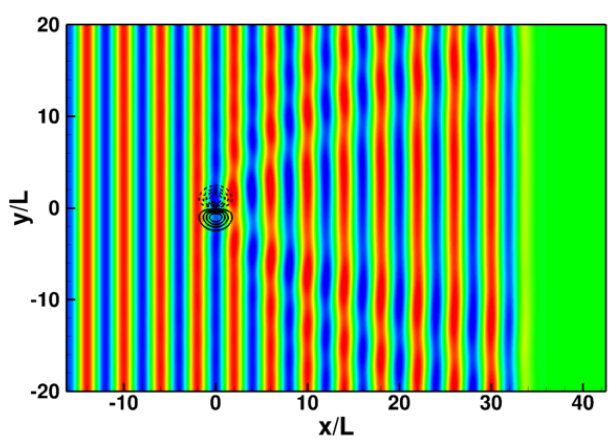

Figure 3: Snapshot of the total pressure fluctuation field $p^{\prime} / P_{i}$ (levels between \pm 1 ). The lines represent the vortex axial velocity (contours between \pm 0.125 , negative values in dashed lines).

A similar calculation is conducted with the modal LEE method. In this case, the vortex core is $L=1$ (and so $\lambda_{0}=4$ ) and the radial direction is discretized over $0 \leq r \leq 15$ with 150 points (leading to approximately 


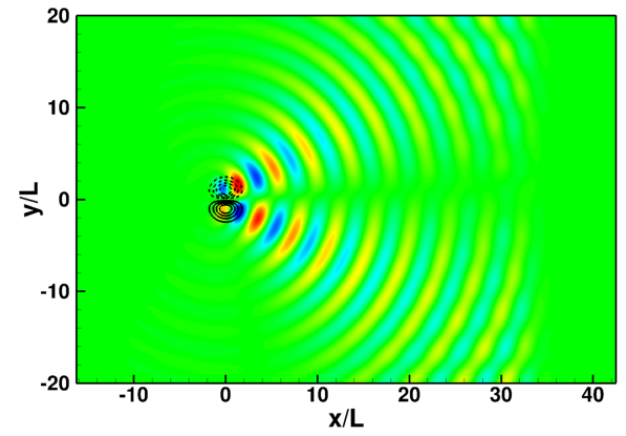

(a)

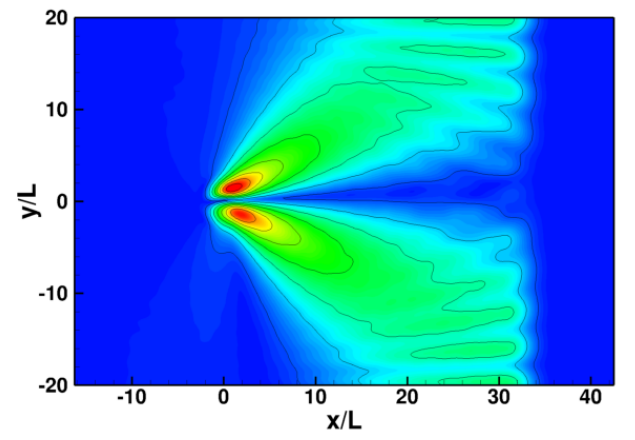

(b)

Figure 4: PIANO results: (a) Snapshot of the scattered pressure fluctuation field $p_{s}^{\prime} / P_{i}$ (levels between \pm 0.25 ). The lines represent the vortex axial velocity (contours between \pm 0.125 , negative values in dashed lines). (b) RMS field of the scattered pressure $p_{R M S}^{\prime} / P_{i}$ (levels between 0 and 0.2 ).

10 points to discretize $L$ ). The system in Eq.(10) is solved for each azimuthal mode between $-30 \leq m \leq 30$ (the decrease of the modal amplitudes is checked in order to ensure that the modes neglected have negligible amplitudes) in order to get $p_{s, m}(r)$ and the scattered pressure field in the $(r, \theta)$ space is constructed:

$$
p_{s}^{\prime}(r, \theta, t)=\sum_{m} p_{s, m}^{\prime}(r) \mathrm{e}^{\mathrm{i}\left(\omega_{0} t-m \theta\right)}
$$

Figures 5(a) and 5(b) present instant scattered pressure (real part) and RMS scattered pressure fields obtained with the modal LEE method. The patterns observed are similar to the results obtained with PIANO (figures 4(a) and 4(b)). The results obtained with these two methods are presented in figure 6 , in terms of directivities over circles located between $r=0.5 \lambda_{0}$ and $r=2.5 \lambda_{0}$ around the vortex $\left(\theta=0^{\circ}\right.$ is aligned with the $x$-direction). The scattered pressure is normalized in order to show that the $1 / \sqrt{r}$ decrease is achieved away from the vortex. One can notice that there is a symmetry between the results of Colonius et al. ${ }^{9}$ and the results of the two methods used in this paper, which has been found to be due to a difference in the spinning direction of the vortex between Colonius et al. and the present results. Apart from this, a very good agreement is found between the two methods and the reference results.

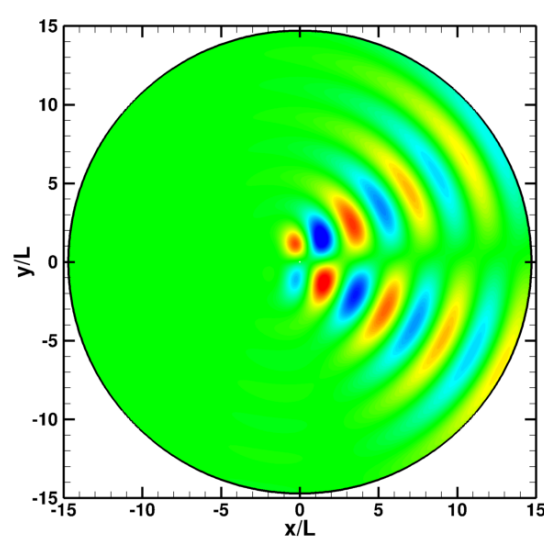

(a)

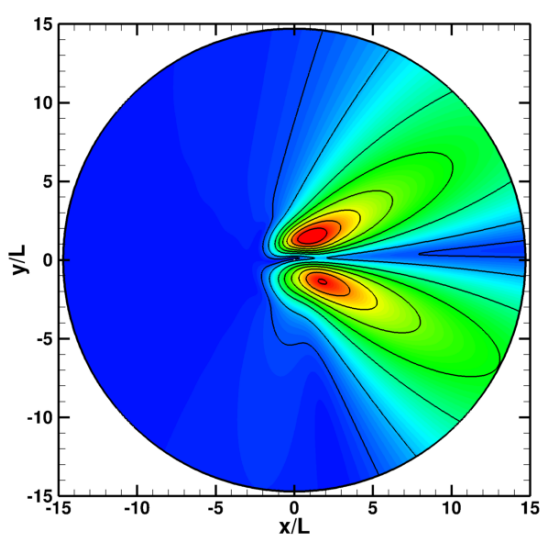

(b)

Figure 5: modal LEE results: (a) Snapshot of the scattered pressure fluctuation field $p_{s}^{\prime} / P_{i}$ (levels between $\pm 0.25)$ and $(b)$ RMS field of the scattered pressure $p_{R M S}^{\prime} / P_{i}$ (levels between 0 and 0.2 ).

The hypothesis of Ewert et al. ${ }^{12,13}$ that the pressure and density fields induced by the vortex have a very low influence on the scattering can be assessed for this case in performing calculations with and without taking these pressure and density fields into account. When they are discarded, only the uniform pressure and density fields remains $\left(\rho=\rho_{\infty}\right.$ and $\left.p=p_{\infty}\right)$. The previous PIANO calculation is then run again 


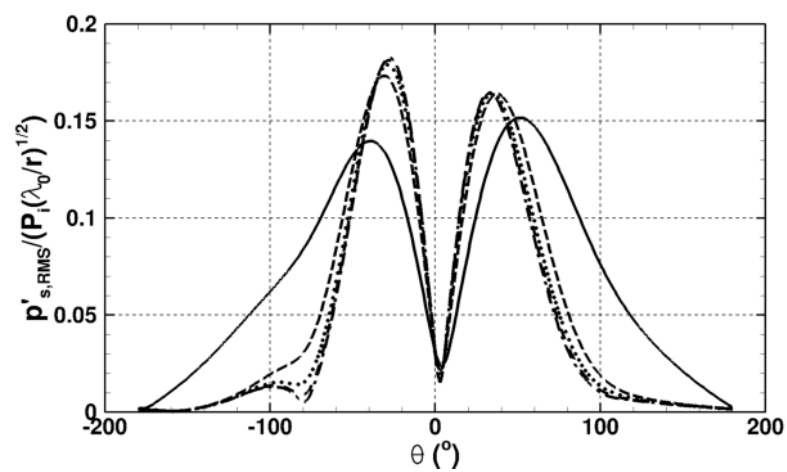

(a)

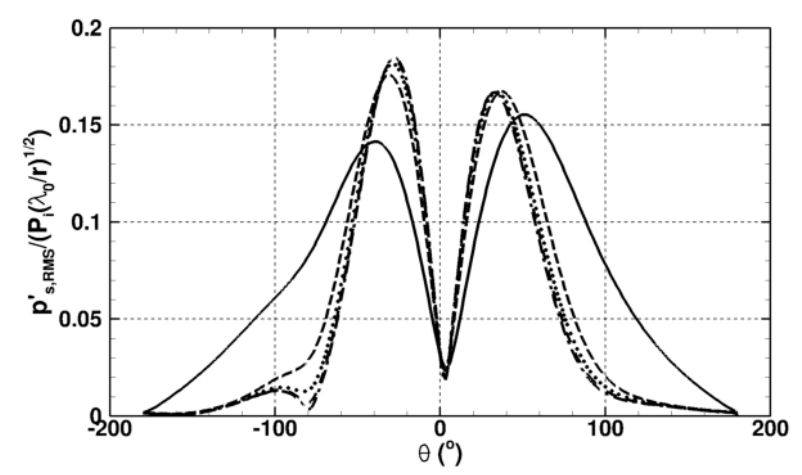

(b)

Figure 6: Comparison between the RMS scattered pressure obtained $(a)$ with PIANO and $(b)$ with the modal LEE method at different radii: $(\longrightarrow) r=0.5 \lambda_{0},(---) r=\lambda_{0},(\cdots \cdots) r=1.5 \lambda_{0},(\boldsymbol{- - \boldsymbol { O }}) r=2 \lambda_{0}$, (-. - $) r=2.5 \lambda_{0}$.

with a base flow including only the velocity field of the vortex, and the directivities resulting from the two calculations are compared over a circle located at $r=2.5 \lambda_{0}$ around the center of the vortex in figure 7 . The two results are very similar, which tend to confirm Ewert et al. hypothesis. As a consequence, the PIANO computations realized in section IV with a convected vortex will only account for the velocity fluctuations induced by the vortex in the unsteady base flow, as it is done by Ewert et al. ${ }^{12,13}$ when solving similar equations.

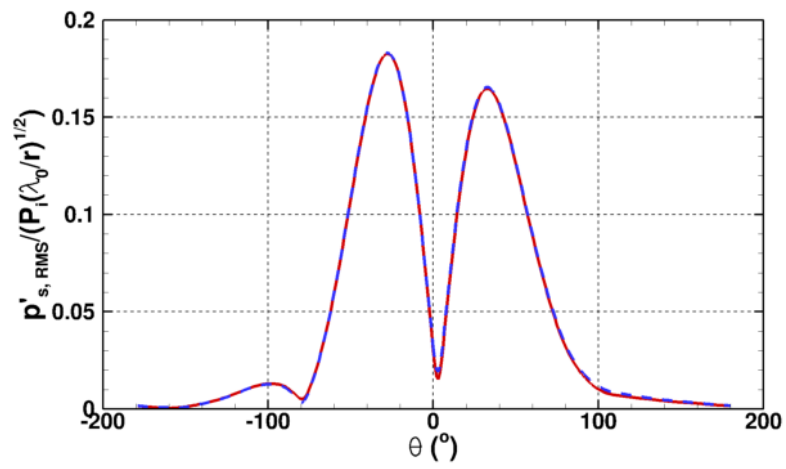

Figure 7: Comparison between the RMS scattered pressure on a circle located at $r=2.5 \lambda_{0}$ around the vortex, ( $(-)$ ) without enforcing the vortex pressure and density fields $\left(\rho_{t}=p_{t}=0\right)$ and $(-\mathbf{-})$ with the pressure and density fields decribed in Eq. (1).

\section{III.B. Effects of the vortex magnitude}

Based on the configuration considered in the previous section, two other computations have been realized using the modal LEE method with $\mathrm{M}_{v}=0.0625$ and $\mathrm{M}_{v}=0.25$ in order to study the effect of the vortex magnitude on the scattering. The directivities at $r=2.5 \lambda_{0}$ for these three cases are plotted in figure 8(a), and they are normalized by their respective maximum in figure $8(\mathrm{~b})$. As expected, increasing the vortex magnitude leads to an increase of the scattered levels. However, a noticeable difference is observable on these directivities by comparison with some analytical models based on low frequency and low Mach number assumptions. ${ }^{9,24}$ These models predicts a symmetrical radiated field about $\theta=0^{\circ}$ and a linear growth of the scattered field with $\mathrm{M}_{v}$. In the present results, we can observe an assymetry on the directivities, which tends to increase when the vortex magnitude is increased as the maximum of the lobe for $\theta<0^{\circ}$ grows slightly faster than $\mathrm{M}_{v}$ and the maximum of the lobe for $\theta>0^{\circ}$ grows slower than $\mathrm{M}_{v}$. The presence of an assymetrical radiation had already been noticed by Colonius et al. ${ }^{9}$ in their numerical study and has been attributed to the effects of terms with an order in $\mathrm{M}_{v}$ higher than the one retained in their analytical model. 


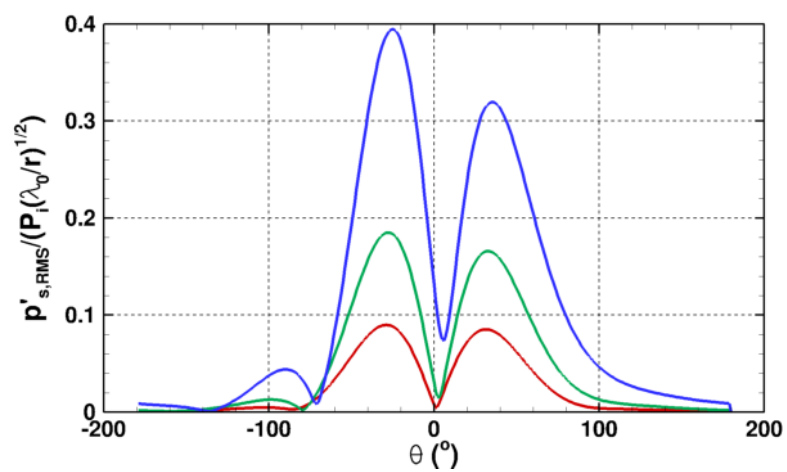

(a)

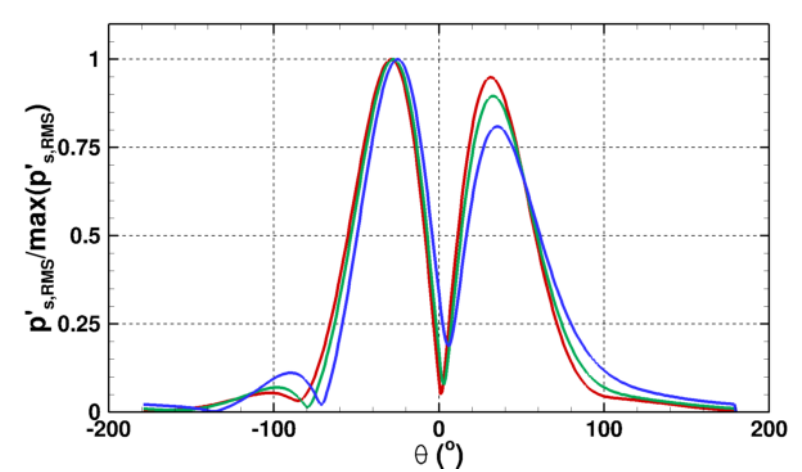

(b)

Figure 8: RMS scattered pressure obtained with different values of $\mathrm{M}_{v}$ at $r=2.5 \lambda_{0}$ around the vortex $(a)$ without normalization and $(b)$ normalized by their maximum values. $(-) \mathrm{M}_{v}=0.0625,(-) \mathrm{M}_{v}=0.125$ and (-) $\mathrm{M}_{v}=0.25$.

\section{III.C. Evolution of the scattered field with the acoustic wavelength}

As the computational cost of the modal LEE method is low, it is possible to perform a set of calculations for a range of incident plane wave frequencies (or Helmholtz numbers, defined as $\mathrm{He}=\omega L / c_{\infty}$ ) in order to draw a cartography of the far-field directivity $D(\theta) / \sqrt{k_{0}}$ (using the expression presented in eq. (14)) as a function of the observation angle $\theta$ and the Helmholtz number. In this section 100 calculations are performed for a vortex with $\mathrm{M}_{v}=0.05$ and Helmholtz numbers of the incident plane wave varying between $\mathrm{He}_{\text {min }}=\pi / 5$ $\left(\lambda_{0}=10 L\right)$ and $\mathrm{He}_{\max }=8 \pi\left(\lambda_{0}=L / 4\right)$. For each case, the discretization of the radial direction ensures 12 points per wavelength when $\lambda_{0} \leq L$ or 12 points to discretize the vortex core $L$ when $\lambda_{0} \geq L$. The number of azimuthal modes summed to get the solutions is also evolving with the incident wave frequency and is up to $-300 \leq m \leq 300$ for the highest frequency. The resulting cartography of the directivity of the scattered pressure is plotted in figure 9 as well as slices for some chosen values of He. Note that the angular range represented in the slices is restricted when He increases in order to discern the directivity lobes properly. We can observe that the intensity of the scattered field increases strongly when the frequency of the incident wave increases (nearly 3 orders of magnitude on the maximum value of the scattered field between the maximum and minimum values of He considered). The shape of the directivity is also drastically changing. For the smallest values of He considered, the directivity is quadripolar, but the backscattering $\left(|\theta|>90^{\circ}\right)$ tends to disappear progressively and only lobes in the forward direction remain at higher He. The number of lobes for $\theta<0^{\circ}$ increases with He while they tend to be more and more concentrated around $\theta=0^{\circ}$, and the main lobe even overlaps $\theta=0^{\circ}$ at high He.

The evolutions of the angular positions and magnitudes of the maxima of the scattered directivity, respectively for $\theta<0^{\circ}$ and $\theta>0^{\circ}$ are plotted on figure 10. Figure 10(a) confirms that the maxima of the directivity get closer to $\theta=0^{\circ}$ when He increases, except between $22<\mathrm{He}<25$ for the $\theta>0^{\circ}$ maximum which seems to move slighly away as the other maximum gets really close to $\theta=0^{\circ}$. Figure 10 (b) shows that the magnitude difference between the $\theta<0^{\circ}$ and $\theta>0^{\circ}$ maxima gets larger when He increases. On this figure, the directivity maximum for $\theta>0^{\circ}$ seems to reach a quasi-constant level at high values of He, while the maximum for $\theta<0^{\circ}$ continues its growth. 


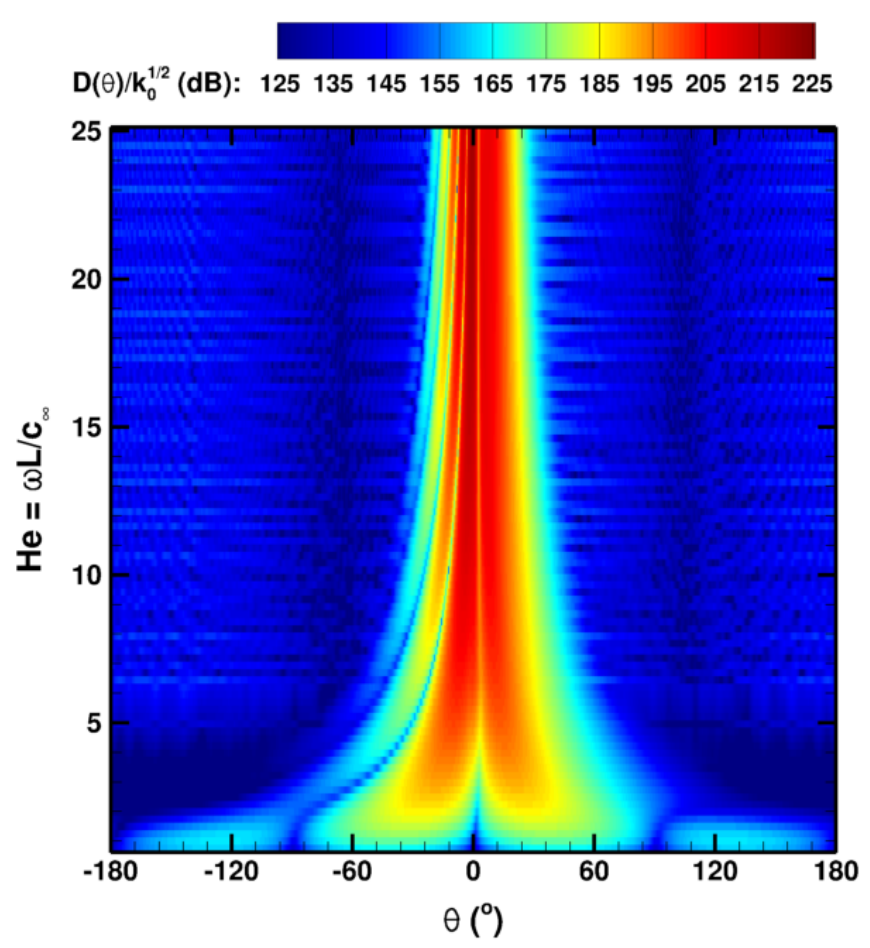

(a)

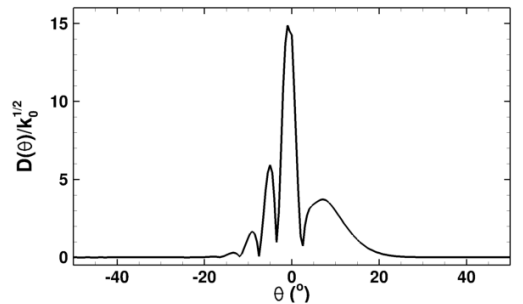

(b) $\mathrm{He}=8 \pi\left(\lambda_{0}=L / 4\right)$

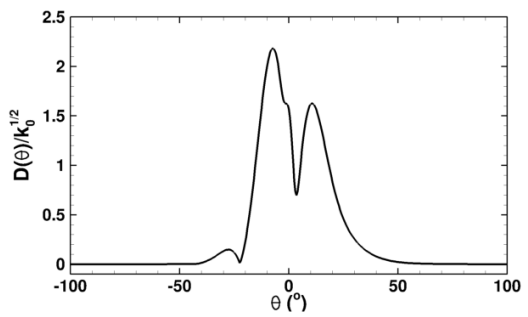

(c) $\mathrm{He}=2 \pi\left(\lambda_{0}=L\right)$

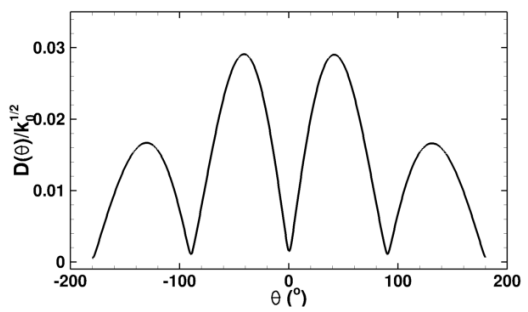

(d) $\mathrm{He}=\pi / 5\left(\lambda_{0}=10 L\right)$

Figure 9: (a) Cartography of the far-field scattered pressure directivity $D(\theta) / \sqrt{k_{0}}$ for a range of Helmholtz numbers. $(b),(c),(d)$ slices for different values of He.

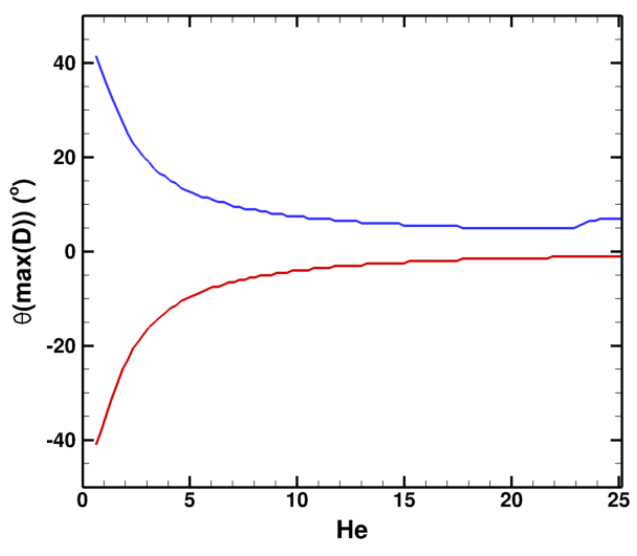

(a)

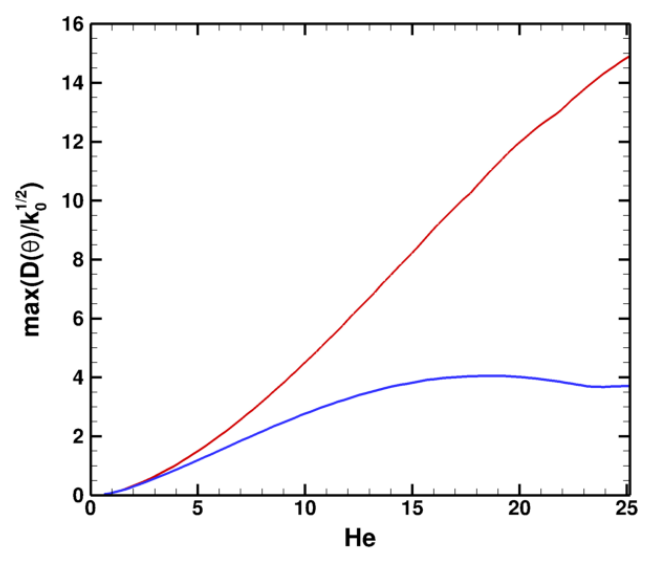

(b)

Figure 10: Evolution of $(a)$ the angular positions and $(b)$ the magnitudes of the maxima of the scattered directivity with the incident wave Helmholtz number for $(-) \theta>0^{\circ}$ and $(-) \theta<0^{\circ}$.

\section{Scattering of an harmonic point source by a convected vortex}

\section{IV.A. Comparison between the two numericals methods for a plane wave}

The two methods are compared on a computational case considering a plane wave propagating in the $+y$ direction, scattered by a vortex convected by a uniform mean flow in the $x$-direction. The wavelength of the plane wave and magnitude of the vortex are similar to the one considered in section III.A $\left(\lambda_{0}=4 L\right.$, 
$\left.\mathrm{M}_{v}=0.05\right)$. The convection velocity is $\mathrm{M}=0.176$. For the modal LEE calculation, the scattering is first estimated by considering a steady vortex, as in section III.A, then the Doppler effects corrections presented in section II.C.2 are accounted for, in order to generate the time signal of the scattered pressure at a microphone position $\left(x_{M}, y_{M}\right)=(0,10 L)$. The numerical domain designed for the PIANO calculation is presented in section IV.B and figure 14, as this case is also part of the parametric study. In PIANO, the vortex velocities $\left(u_{t}, v_{t}\right)$ are introduced once the acoustic field is well established in the domain. The initial position of the vortex center is slightly upstream of the inflow boundary in order to smoothly introduce the vortex in the domain. The time signal is recorded during the whole traversal of the vortex.

A snapshot of the scattered pressure (reduced by the amplitude of the incident radiation alone at the central microphone location $P_{i}$ ) for the PIANO calculation is presented in figure 11 at the time when the vortex is in the middle of the computational domain. The scattered field is very similar to what has been observed for a stationnary vortex in section III.A, except that this scattered field is moving in the $x$-direction with time. The time signals recorded by the microphone are presented in figure 12 for both methods. The time $t=0$ corresponds to the position $x_{v}=0$ of the vortex center (same $x$-position than the microphone). The two signals have a very similar shape and the passages of the two beams corresponding to the two main scattering directions (visible in figure 11) give rise to the two humps observable on the time signals. The low scattered pressure levels around $t=0$ are due to the quasi-absence of scattering around the direction of propagation of the plane wave (also visible in figure 11). The SPL of these signals are plotted in figure 13(a) as a function of the Strouhal number St $=f L / u_{0}$, and the levels are scaled by the amplitude of the incident wave. For the PIANO computation, the SPL of the total pressure fluctuation, including the incoming wave, is plotted in order to see the peak at the incident wave frequency. The SPL obtained with the two methods are in very good agreement. They show the appearance of two sidebands surrounding the peak, that can be linked to the two scattered beams. Due to the Doppler effect induced by the movement of the vortex relative to the microphone, the passage of the first beam (when the vortex is upstream of the microphone) gives rise to the high frequency sideband, and the passage of the second beam (when the vortex is downstream of the microphone) gives rise to the low frequency sideband. This is demonstrated in figure 13(b) where SPL are calculated after splitting the time signal at $t=0$. Even if it has been seen in section III.B that the scattering is not symmetrical about the plane wave propagation direction, the asymmetry is slight for the value of $\mathrm{M}_{v}$ chosen in this case. As a consequence, the amplitude of the two sidebands are nearly identical (only a 0.125 $\mathrm{dB}$ difference).

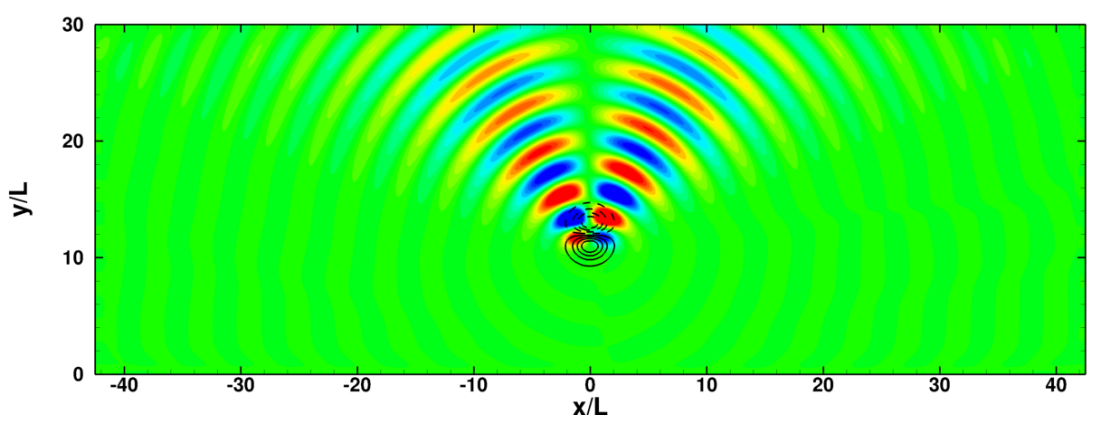

Figure 11: Snapshot of pressure scattered by a convected vortex for an incident plane wave propagating in the $+y$-direction. Color levels: $p_{s}^{\prime} / P_{i}$ between \pm 0.075 and lines: vortex axial velocity between \pm 0.05 . 


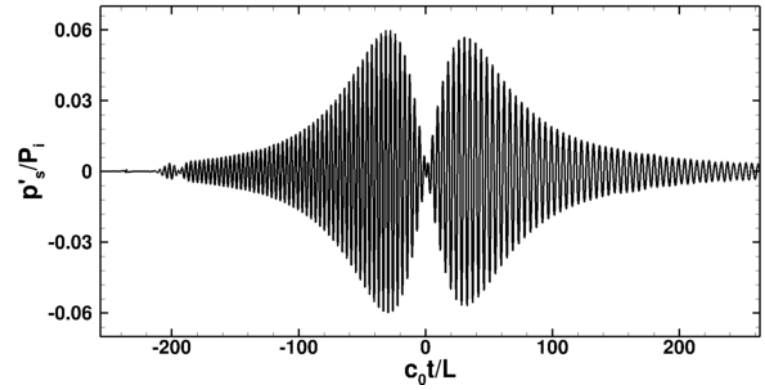

(a) PIANO

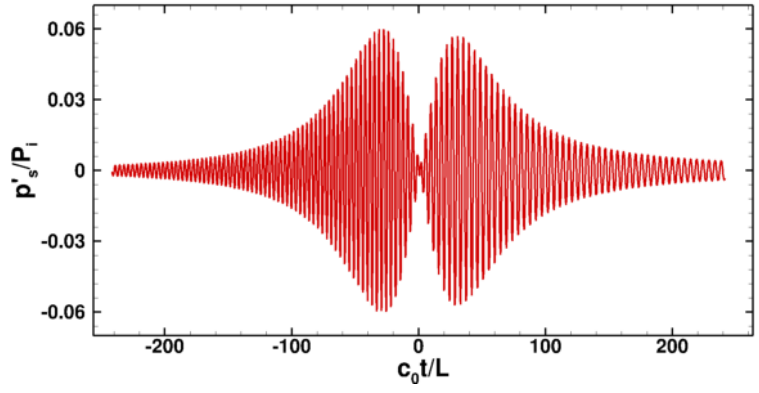

(b) modal LEE

Figure 12: Time signals of the scattered pressure recorded for the scattering of a plane wave by a convected vortex.

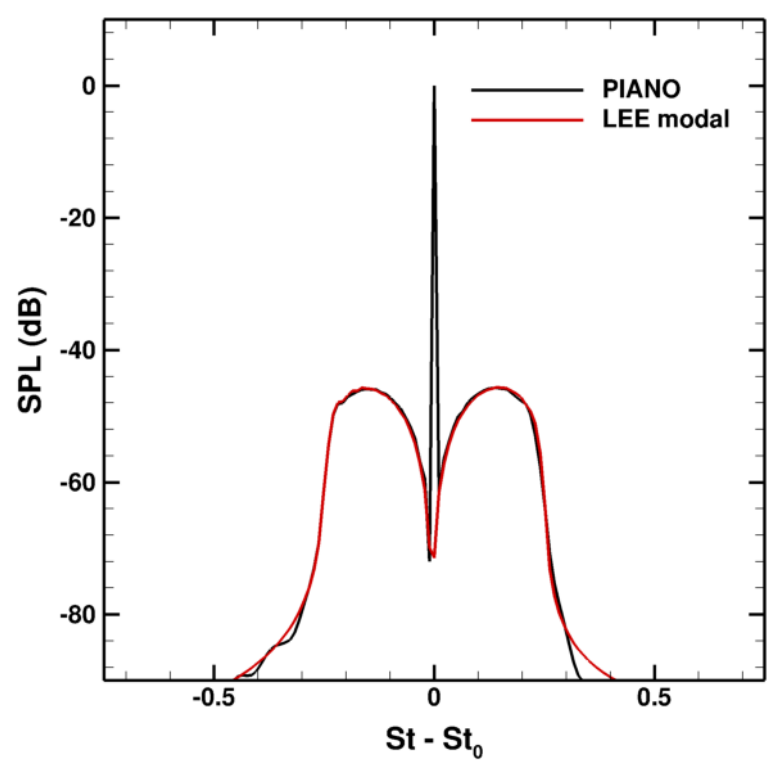

(a)

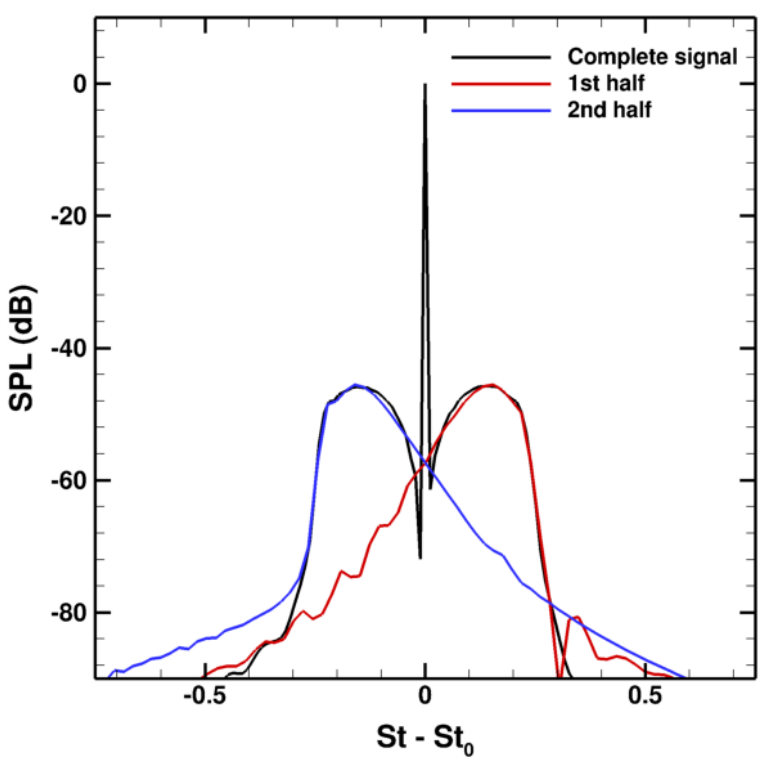

(b)

Figure 13: SPL of the scattered pressure recorded by the microphone for the scattering of a plane wave by a convected vortex. (a) Comparison between PIANO and the modal LEE method. (b) SPL of the time signal splitted at $t=0$.

\section{IV.B. Results of the parametric study realized with PIANO}

A parametric study has been conducted on the scattering of a monopole radiation by a convected vortex, focusing on 4 parameters: the acoustic source wavelength (based on $\lambda_{0}=c_{0} / f_{0}$ ), the convection velocity, the vortex magnitude and the distance between the source and the vortex path. Three values are chosen for each parameter, except for the source wavelength where five values are considered. Table 1 describes all the cases considered and the corresponding values of the parameters. The bold column in the table is the baseline case that will be used in every comparison. The case where the distance between the source and the vortex path is $d=\infty$ corresponds to the plane wave already presented in section IV.A. For each case, the calculation is run with and without the vortex in order to compute the scattered field. A schematic of the computational domain used to performed this study is presented in figure 14. The vortex core is discretized by 10 points with cell sizes $\Delta x=\Delta y=1$ leading to $851 \times 301$ points in the domain. For the two smallest values of the acoustic wavelength, specific computational domains have been designed with reduced dimensions in the $y$-direction in order to limit the computational effort. For these two cases, the size of the 
vortex is increased in order to ensure that the acoustic waves are discretized by 12 points per wavelength in any direction while keeping the same cell size. The incoming sound field (monopole or plane wave) is injected in the domain through a 20 points wide sponge layer adjacent to the bottom boundary. On the other boundaries, Tam's radiation boundary condition ${ }^{27}$ is applied when the incident radiation considered is a monopole, and Thompson's $1-\mathrm{D}^{28}$ characteristic condition when it is a plane wave. An additionnal 50 points dissipative sponge layer is placed next to the upper boundary. The microphone signals used to compute the SPL spectra are recorded between the time the vortex enters the domain and the time it leaves the domain.

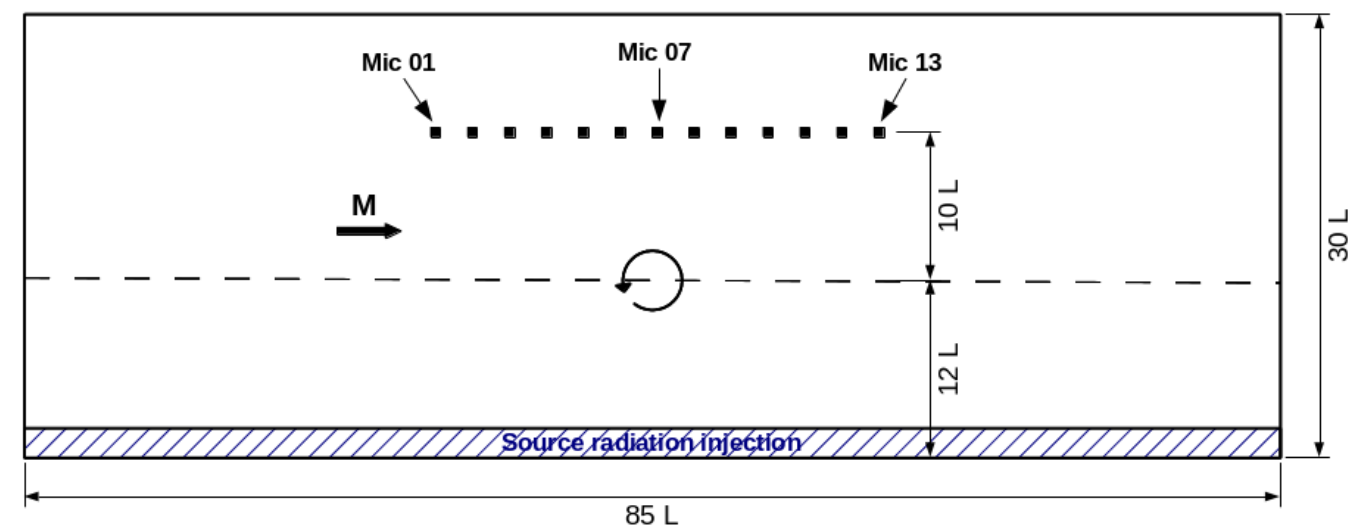

Figure 14: Schematic of the computational domain considered for the parametric study with PIANO.

\begin{tabular}{|l||c|c|c|c|c|c|c|c|c|c|c|}
\hline$\lambda_{0}$ & $L / 2$ & $1 L$ & $2 L$ & $4 L$ & $\mathbf{4 L}$ & $4 L$ & $4 L$ & $4 L$ & $4 L$ & $4 L$ & $8 L$ \\
$\mathrm{M}$ & 0.176 & 0.176 & 0.176 & 0.088 & $\mathbf{0 . 1 7 6}$ & 0.352 & 0.176 & 0.176 & 0.176 & 0.176 & 0.176 \\
$\mathrm{M}_{v}$ & 0.05 & 0.05 & 0.05 & 0.05 & $\mathbf{0 . 0 5}$ & 0.05 & 0.025 & 0.1 & 0.05 & 0.05 & 0.05 \\
$d$ & $20 L$ & $20 L$ & $20 L$ & $20 L$ & $\mathbf{2 0 L}$ & $20 L$ & $20 L$ & $20 L$ & $40 L$ & $\infty$ & $20 L$ \\
\hline $\mathrm{St}_{0}$ & 11.36 & 5.68 & 2.84 & 2.84 & $\mathbf{1 . 4 2}$ & 0.71 & 1.42 & 1.42 & 1.42 & 1.42 & 0.71 \\
\hline
\end{tabular}

Table 1: Parameters of the different cases considered for the parametric study. The case in bold is the baseline case.

\section{IV.B.1. Effects of the distance between the source and the vortex path}

In this section, the distance between the monopole source and the vortex path is increased from $d=20 L$ (reference case) to $d=40 L$ and finally $d=\infty$ (the plane wave case). Snapshots of the scattered field for these three cases at different times are presented in figure 16.

The study of a single vortex is particularly interesting here, because it hightlights noticeable differences in the scattering directions between the monopole cases and the plane wave, depending of the vortex position. The two main scattering directions can be observed on the three cases, but depending on the position of the vortex, the orientation of the scattered field evolves in relation with the propagation direction of the monopole wavefronts. When the source distance is increased, the wavefronts curvature is reduced (they are perfectly straight for the plane wave) and, as a result, the scattered field is less tilted towards the upstream (or downstream) direction when the vortex is upstream (or downstream). We can observe this change between $d=20 L$ and $d=40 L$, and ultimately for the plane wave case where the scattered field keep the same orientation during the complete vortex traversal.

The SPL for these three cases, at the central microphone position, are plotted in figure 15(a). The spectra for the two monopole cases are relatively similar. The sidebands appear wider for the monopole cases than for the plane wave case, especially the high frequency sideband, and secondary sidebands are visible for the monopole cases. These secondary sidebands can be attributed to the scattering by the vortex when it is far from the source in the $x$-direction. When the vortex is far, the main scattered radiation is strongly oriented towards the upstream or downstream direction and as a result, some secondary lobes visible on 
the directivity for high radiation angles (see figure 8 for example) will be radiated towards the microphone during a short time. Because of the large distance in the $x$-direction during these events, the angle between the vortex and the microphone is very small and so the Doppler shift is important, which explains why these secondary sidebands are further away from the tone. Slight differences can also be observed between the spectra at different microphone positions for the reference case $(d=20 L)$ in figure $15(\mathrm{~b})$. The shapes and levels are not exactly the same because of the evolution of the scattered field with the vortex position. For example, the convective amplification of the monopole radiation towards the upstream directions leads to higher scattered pressure fluctuations in this region, and the levels observed for the first microphone are slightly higher than for the others. Also, the changes in the wavelength of the incident radiation depending on the radiation direction (because of the mean flow) lead to changes in the scattering pattern with the position of the vortex, and thus to changes on the SPL.

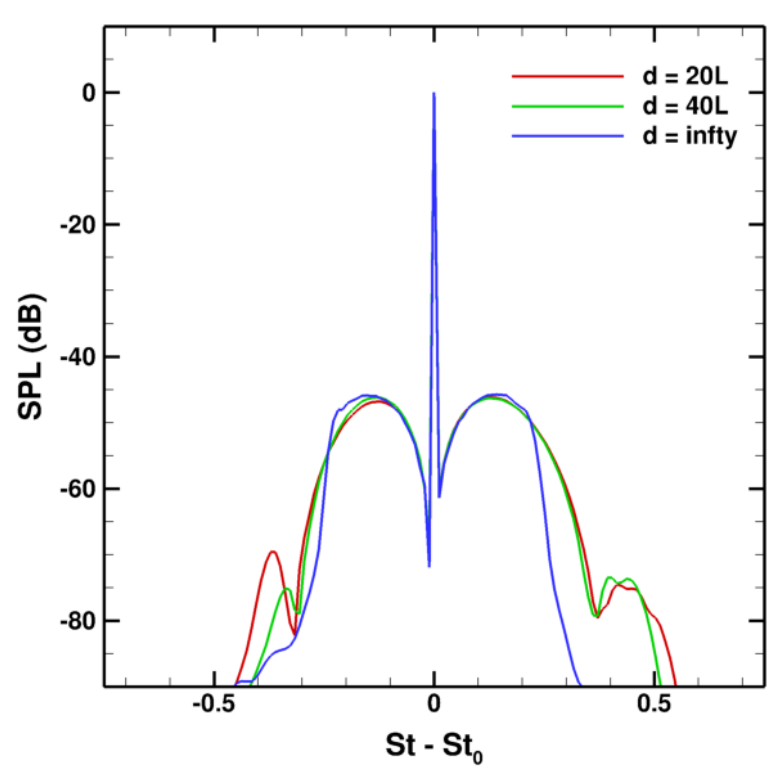

(a)

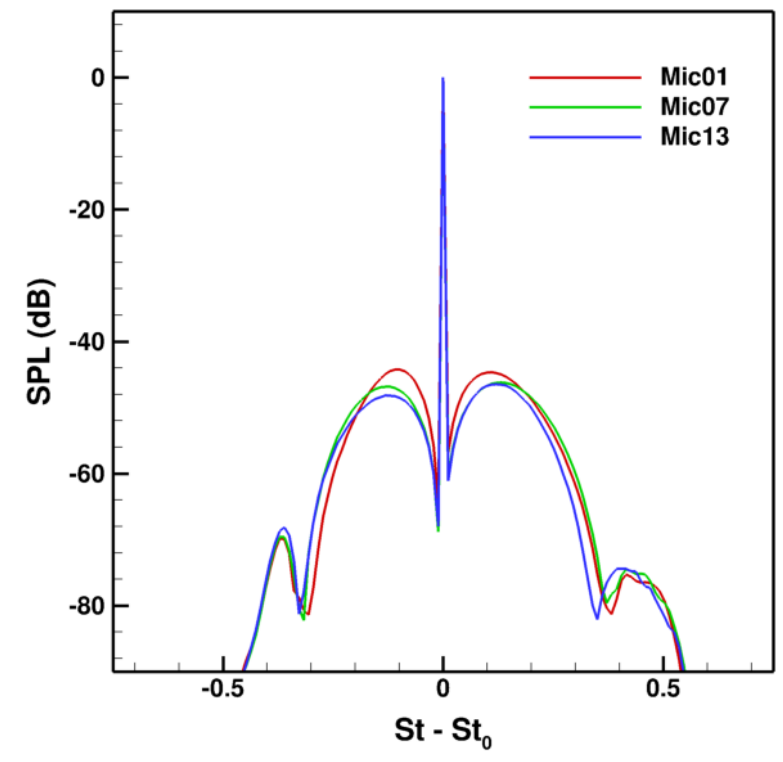

(b)

Figure 15: Effects of the distance between the source and the vortex path. (a) SPL at the central microphone location for the different source distances $(b)$ SPL at different microphone locations for the reference case $(d=20 L)$. 

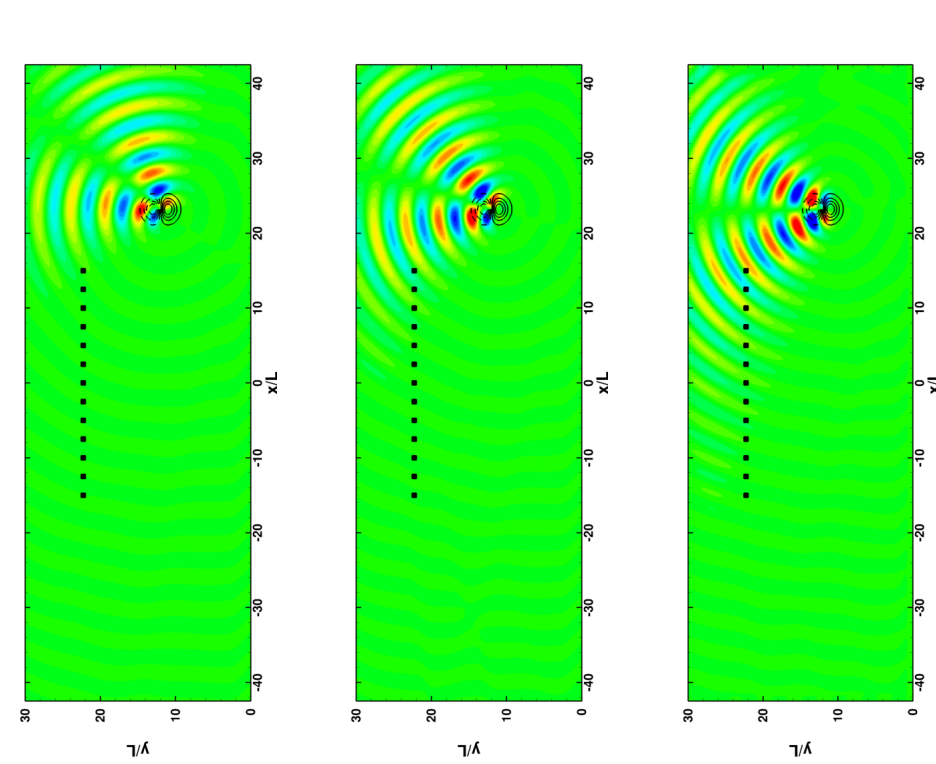

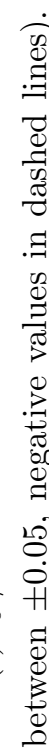
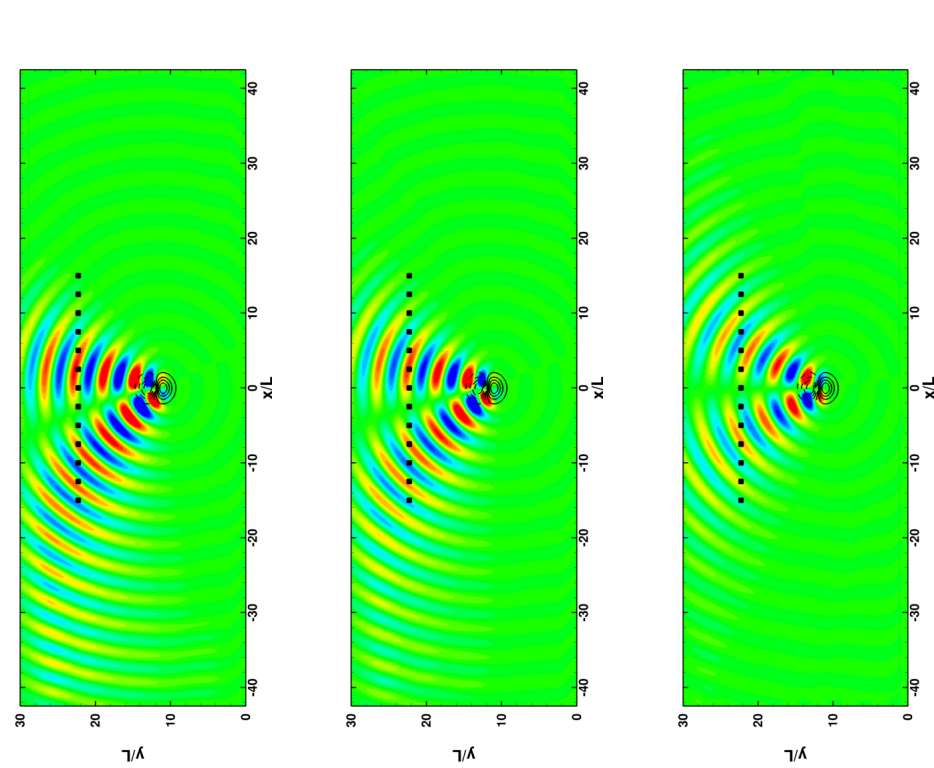

$\frac{0}{2}$

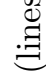

莺

$\stackrel{0}{8}$

. స్తే

嵌

용

궁

อิ
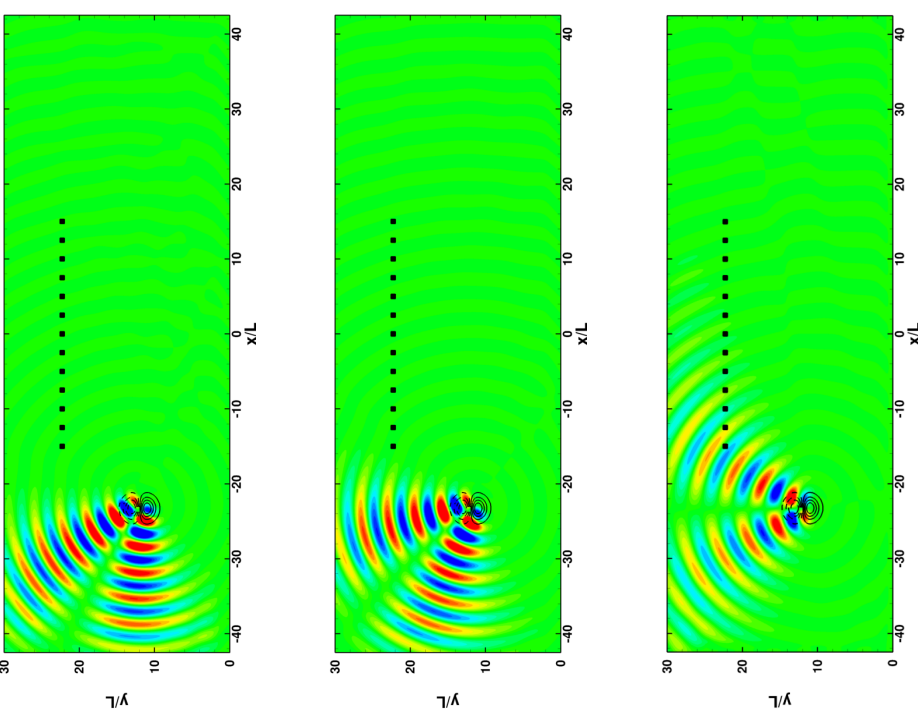

H

콩

苋苦

远

$\pi 8$

己

훙

递

을

$-2^{2} \cdot$

잉

।

$\|$ 常

苍 क्ञ (త)

옹

点

16 of 23 


\section{IV.B.2. Effects of the source wavelength}

In this section, the acoustic wavelength is increased from $\lambda_{0}=L / 2$ to $\lambda_{0}=8 L$. Snapshots of the scattered pressure are presented for the five cases considered in figure 17 , confirming the observation made in section III.C for the steady vortex that the angular width of the scattering beam decreases when the wavelength is decreased. On the contrary, for the largest wavelength, the scattered radiation exhibits a quadripolar pattern. The scattered levels are higher for smaller wavelengths, which also corresponds to observations made in section III.C. This increase of the scattering for shorter wavelength is also coherent with observations made in previous studies on the scattering by a turbulent shear layer. ${ }^{7,13,20,22,26}$

The signals from the central microphone are presented in figure 18. Due to the widening of the scattering beam when the wavelength is increased, the time range where the scattering is visible on the recorded signals becomes more important. Thus, the time intervals represented for figures (a) to (d) are restricted in order to have a better view of the scattering effect, whereas the complete vortex traversal time is plotted in figure (e) since scattered pressure fluctuations are visible during the whole calculation for this case. The difference in the scattered levels is clearly visible in these figures, with a difference of nearly two orders of magnitude between the smallest and the largest wavelengths. As for the plane wave case discussed in section IV.A, most of the time signals present two humps, except for $\lambda_{0}=8 L$ where two additional humps are visible because the quadripolar pattern leads to scattered waves emitted towards the microphone when the vortex is far upstream or downstream (similarly to what is discussed in section IV.B.1). Some differences appear between the two main humps in terms of length and intensity because of the assymmetry of the scattering (see section III.C) and because of the evolution of the scattering radiation with the vortex position (see section IV.B.1).

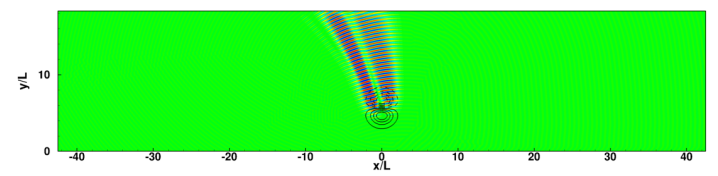

(a) $\lambda_{0}=L / 2, p_{s}^{\prime} / P_{i}$ levels between \pm 1.05

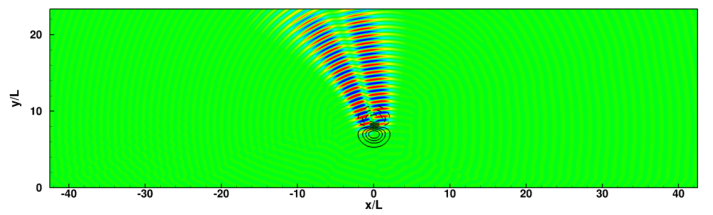

(b) $\lambda_{0}=L, p_{s}^{\prime} / P_{i}$ levels between \pm 0.5

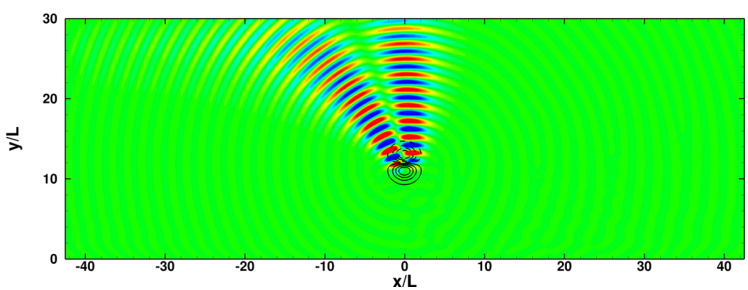

(c) $\lambda_{0}=2 L, p_{s}^{\prime} / P_{i}$ levels between \pm 0.21

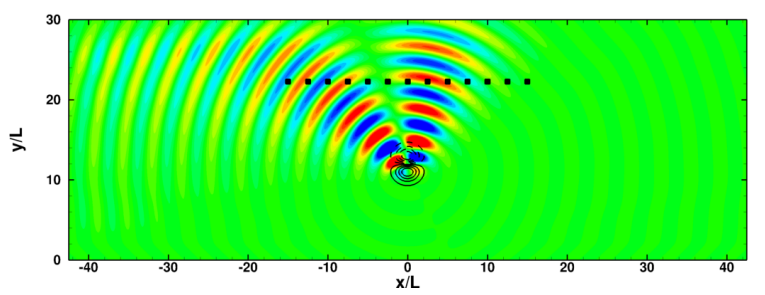

(d) $\lambda_{0}=4 L, p_{s}^{\prime} / P_{i}$ levels between \pm 0.075

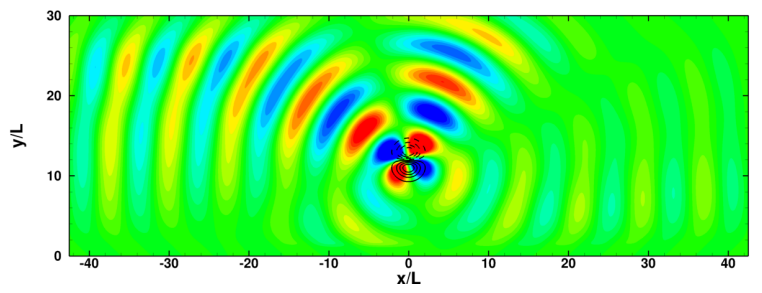

(e) $\lambda_{0}=8 L, p_{s}^{\prime} / P_{i}$ levels between \pm 0.02

Figure 17: Snapshots of the scattered field for different values of the wavelength $\lambda_{0}$. Color levels: reduced pressure $p_{s}^{\prime} / P_{i}$ (see legends) and lines: axial velocity between \pm 0.05 .

The SPL for the different wavelength are plotted in figure 19(a) as a function of the Strouhal number and in figure 19(b) as a function of the reduced Strouhal number at the central microphone position. Figure 19(b) shows that, relatively to the incident frequency, the sidebands are narrower when the wavelength is decreased. This observation is coherent with the narrowing of the scattered beam observed in figure 17 leading to a less important Doppler shift on the spectra.

The previous studies on the scattering by a turbulent shear layer mentioned above observed that the source frequency mainly affects the levels and the frequency decay rate of the sidebands on the spectra, but has few effect on the position of their maxima or on their shape. The spectra display a monotonic decrease away from the peak and the sidebands maxima. In the present study, the increase of the scattered levels 


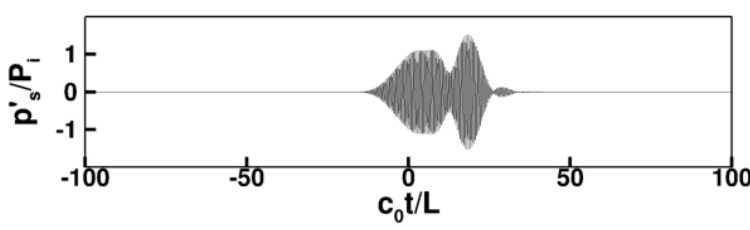

(a) $\lambda_{0}=L / 2$

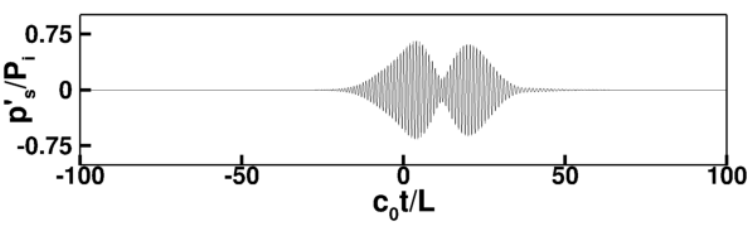

(b) $\lambda_{0}=L$

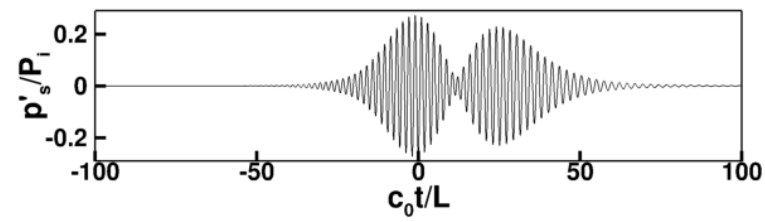

(c) $\lambda_{0}=2 L$

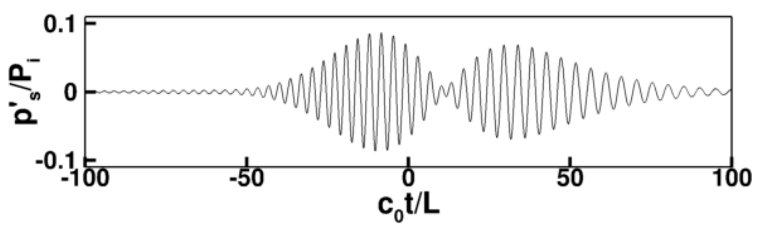

(d) $\lambda_{0}=4 L$

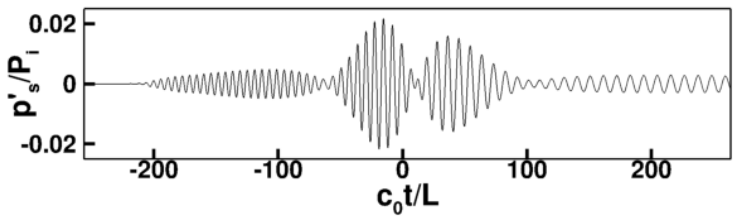

(e) $\lambda_{0}=8 L$

Figure 18: Time signals of the scattered pressure recorded at the central microphone position for different values of the wavelength $\lambda_{0}$.

when $\lambda_{0}$ decreases is visible again in figure 19(a), but other changes are also noticeable on the shape of the spectra. For the largest value of $\lambda_{0}$, the secondary sidebands already mentioned in section IV.B.1 are important relative to the main sidebands because of the strong quadripolar scattering pattern. For small values of $\lambda_{0}$, only one sideband is present at low frequency, but multiple sidebands appear at high frequency. This behavior can be understood by looking at the directivity for a small wavelength in figure 9 . On this figure, we can observe that only one lobe is present for $\theta>0^{\circ}$, but several lobes appear for $\theta<0^{\circ}$. Each of these lobes gives rise to a sideband on the spectrum in figure 19(a) with a different frequency shift. We can even see that the main lobe can be overlapping $\theta=0^{\circ}$, and thus the sideband associated will also overlap the peak frequency, as it is the case here for $\lambda_{0}=L / 2$. These changes with the source frequency might not be visible for a turbulent shear layer, because it can be seen as a large number of eddies with different sizes that are scattering together, leading to an overall scattering that is more or less monotonic compared to the scattering by eddies of a specific size.

Finally, the levels of the peak on the spectra are weakly affected by the scattering for all the acoustic wavelengths considered. For the smallest wavelength, where the scattering is more intense, the amplitude of the peak is only reduced by about $0.3 \mathrm{~dB}$. This is explained by the fact that the traversal of a single vortex produces a finite energy scattering signal with a duration that becomes shorter when the wavelength is decreased (as observed in figure 18), and the SPL are computed from signals covering the whole traversal duration. For a large wavelength, the scattering is observed during the whole traversal but it is very weak, so the peak level is not affected. For a small wavelength the scattering is very intense but only during a short period regarding the whole signal, so its effect is weak on the level of the peak. 


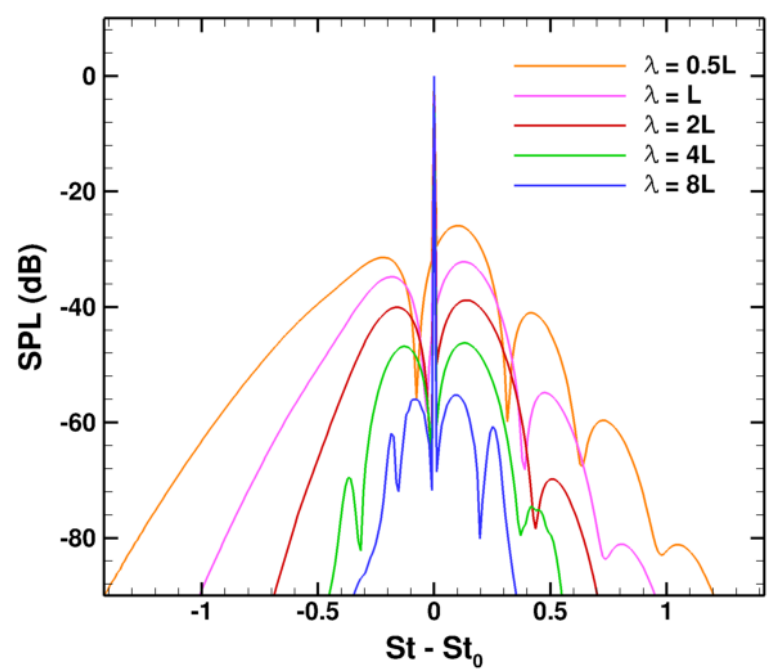

(a)

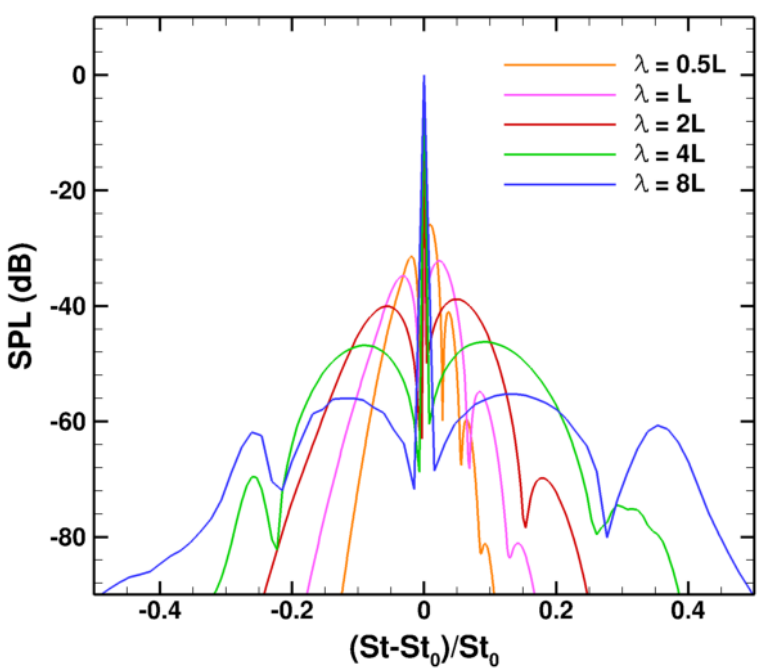

(b)

Figure 19: SPL of the scattered field at the central microphone location for the different values of $\lambda_{0},(a)$ as a function of the Strouhal number and $(b)$ as a function of the reduced Strouhal number.

\section{IV.B.3. Effects of the convection velocity}

The convection velocity is expected to have an important effect on the scattering, since the Doppler effects considered in section II.C.2 are directly linked to the convection velocity. Here, as the source is a monopole, the convection will also affect the propagation of the incoming wavefronts and thus the orientation of the main scattering directions during the vortex traversal. This effect is visible on the snapshots of the scattered field presented in figure 20 where the scattered field is more oriented towards the upstream direction for $\mathrm{M}=0.352$ than for $\mathrm{M}=0.088$. The SPL plotted in figure 21(a) as a function of the Strouhal number are very similar for the three values of the convection velocity considered. The slight differences that can be observed on these spectra are probably due to the convection effect on the incident field mentioned before (similar computations have been performed with a plane wave for several Mach numbers, and the SPL plotted as a function of the Strouhal number are almost perfectly identical). As the Strouhal number defined is normalized by the convection velocity, the similarity of the spectra is a good indicator on the fact that the frequency shift of the sidebands and the convection velocity are directly related. The effect of changing the convection velocity can be seen more clearly if the same SPL are plotted as a function of the frequency as in figure 21(b). On this figure, we clearly see that when the convection velocity is increased, the sidebands become wider. A similar observation is also made for the spectral broadening by a turbulent shear layer in several studies existing in the literature. ${ }^{7,13,20,22,26}$ 


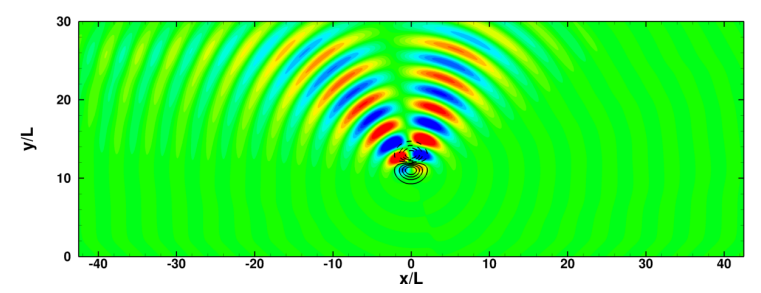

(a) $\mathrm{M}=0.088$

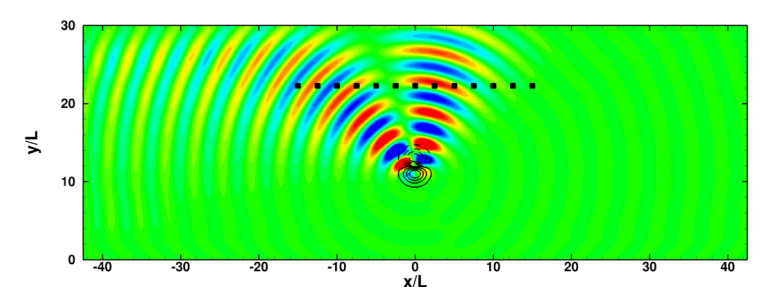

(b) $\mathrm{M}=0.176$

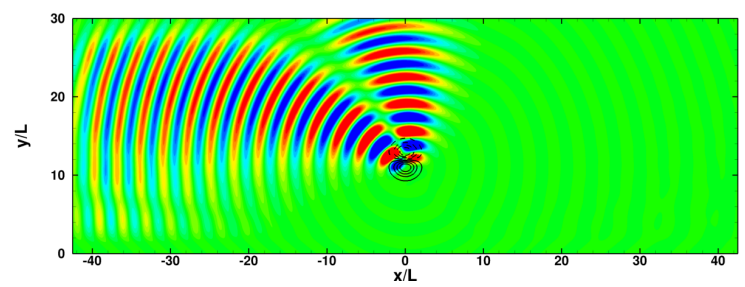

(c) $\mathrm{M}=0.352$

Figure 20: Snapshots of the scattered field for different values of the convection velocity. Color levels: $p_{s}^{\prime} / P_{i}$ between \pm 0.075 and lines: axial velocity between \pm 0.05 .

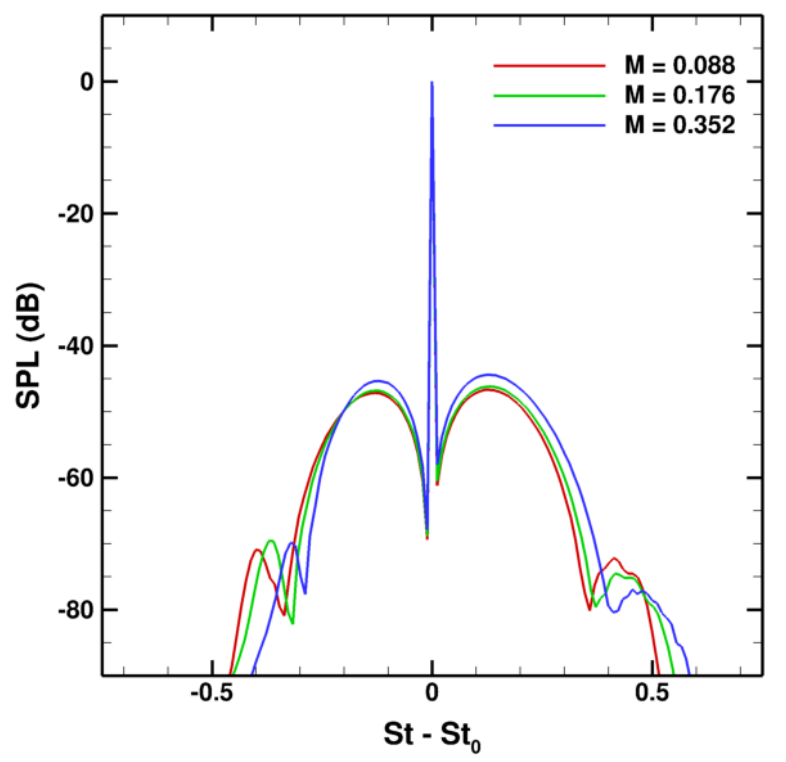

(a)

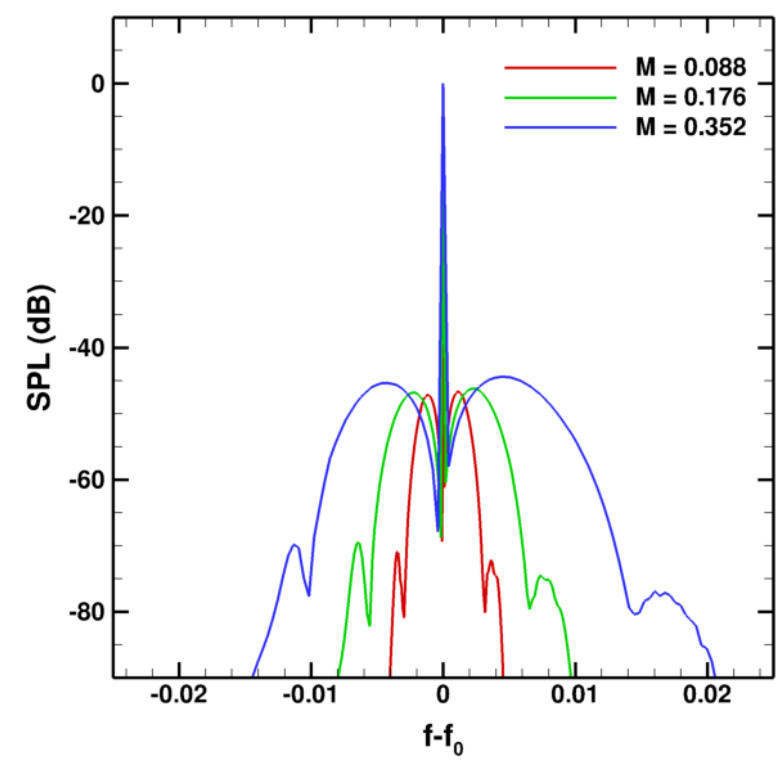

(b)

Figure 21: SPL of the scattered field at the central microphone location for different values of the convection velocity. 


\section{IV.B.4. Effects of the vortex magnitude}

The last parameter studied is the magnitude of the vortex. We have seen in section III.B that increasing the vortex magnitude tends to increase the radiation on one side of the directivity of the scattered field more than on the other (for an incident plane wave). The SPL calculated at the central microphone location for the three values of $\mathrm{M}_{v}$ considered are plotted in figure 22(a). On these SPL, apart from a quasi linear increase of the levels, no drastic changes appear on the main sidebands from one case to an other. The levels of these spectra are normalized by the vortex magnitude in figure 22(b) and shows only some slight changes on the high frequency secondary sideband, which seems to grow more than linearly and to move slightly closer to the peak when the vortex magnitude increases. This behavior might be linked to the change visible in the $\theta<0^{\circ}$ secondary lobe in figure 8 . The range of vortex magnitudes studied here might not be wide enough to reveal changes on the main sidebands.

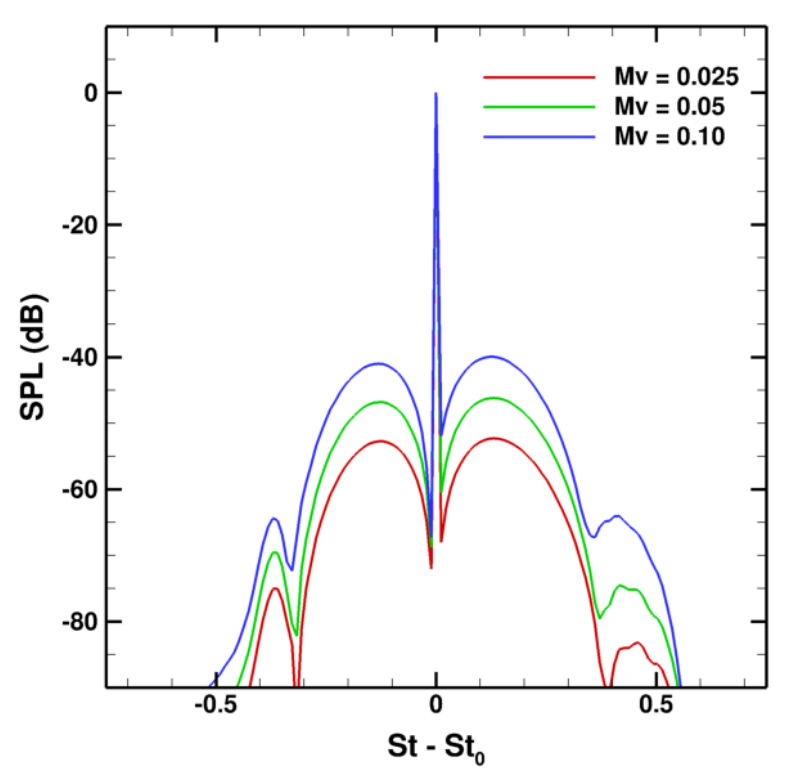

(a)

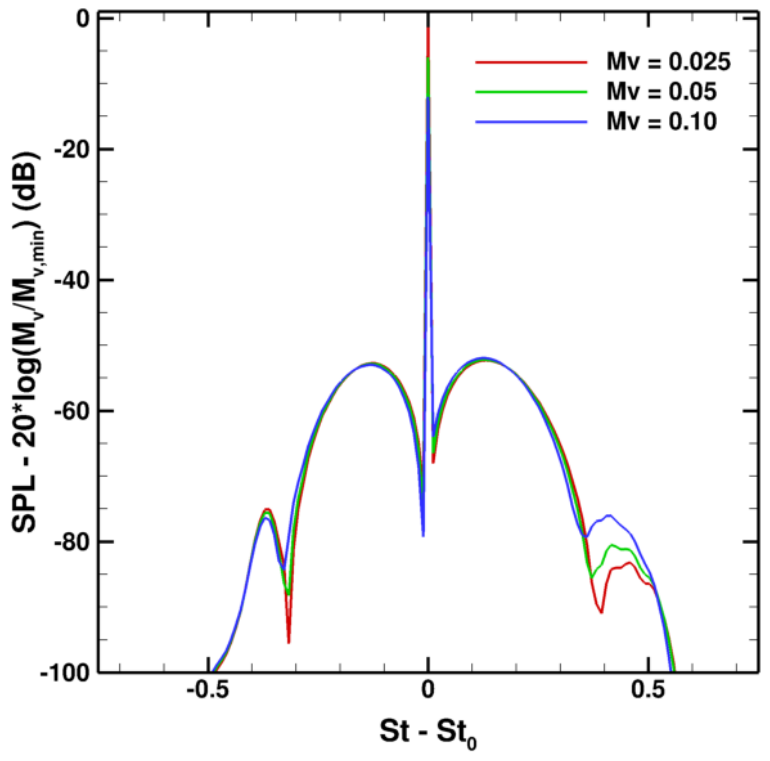

(b)

Figure 22: SPL of the scattered field at the central microphone location for different values of the vortex magnitude.

\section{Conclusion}

The scattering of an harmonic acoustic radiation by a single vortex has been studied in this paper in order to get insight on the more complex problem of the scattering by a turbulent layer including a wide variety of vortex sizes. The choice of numerical methods to perform this study allows to avoid restrictions on the flow or on the frequency of the source that are inherent to analytical models.

If the vortex considered is steady, a time efficient semi-numerical method based on the linearized Euler equations in the frequency domain has been developed and used to study the spatial scattering of a plane wave over a wide range of frequencies. The results show drastic changes in the shapes and levels of the scattered field between the low and high frequency domains. The transition between these two domains can also be observed, whereas most of the analytical model are based either on low or high frequency assumptions for the incident sound field. In addition, when the incident frequency considered approaches the low-frequency limit of several analytical models, the numerical method is able to capture an asymmetry in the scattered field which is not predicted by these analytical methods. This semi-numerical method can be adapted to study the spectral scattering induced by the convection of the vortex in a uniform mean flow by using corrections based on the Doppler effect, but for an incident plane wave only.

For different acoustic sources (i.e. monopole sources) another numerical method is used in this study, 
based on a linearized Euler equations solver in the time domain. In order to capture the interaction between the convected vortex and the acoustic fluctuations, the vortex is introduced as an unsteady component on the base flow field. This method has been used to study the influence of the source frequency, the convection velocity, the vortex magnitude and the source-vortex distance on the scattering. All the spectra obtained in this study display two main sidebands surrounding a peak at the source frequency. This phenomenon is relatively similar to the haystacking observed in previous studies related to the spectral broadening of an harmonic source by a turbulent shear layer. Some of the trends deduced from this study, especially concerning the source frequency and the convection velocity, are also in agreement with the observations made for haystacking. In addition, the present study has provided further details on the spatial and the spectral evolutions of the scattering of a sound field by vortices of a specific size, whereas a turbulent shear layer contains a wide range of eddy sizes. The shape of the sidebands observed on the scattered spectra seem coherent with an explanation based on the spatial scattering by the vortex associated to a Doppler effect.

The next step of this work on spatial and spectral scattering is to replace the single vortex by a turbulent layer synthesized using a stochastic method. Stochastic methods allow to generate turbulent fields with chosen spectra or correlations. It is then possible to change the range of eddy sizes present in the turbulence and the position of the maximum of the turbulence spectra. In a first time, a simple configuration consisting in a turbulent shear layer with constant thickness, convected in a uniform mean flow will be considered. The parameters of the source, the flow and the turbulent layer will be studied in order to deduce some further insight regarding the scattering.

\section{Acknowledgments}

This work was partly supported by Rolls-Royce plc through the University Technology Center in Gas Turbine Noise at the University of Southampton and funded by EPSRC Grant EP/K017551/1.

The authors would like to acknowledge the members of the AS/TA department of DLR-Braunschweig for providing the PIANO solver and the technical support associated.

\section{References}

${ }^{1}$ Bailly, C. \& Juvé, D., "Numerical solution of acoustic propagation problems using Linearized Euler Equations", AIAA Journal, 38(1), 2000, pp. 22-29.

${ }^{2}$ Brown, E., "Turbulent spectral broadening of backscattered acoustic pulses", Journal of the Acoustical Society of Amer$i c a, \mathbf{5 6}(\mathbf{5})$, 1974, pp. 1398-1406.

${ }^{3}$ Brown, E. \& Clifford, S., "Spectral broadening of an acoustic pulse propagating through turbulence", Journal of the Acoustical Society of America, 54(1), 1973, pp. 36-39.

${ }^{4}$ Brown, E. \& Clifford, S., "On the attenuation of sound by turbulence", Journal of the Acoustical Society of America, 60(4), 1976, pp. 788-794.

${ }^{5}$ Campos, L., "The spectral broadening of sound by turbulent shear layers. Part 1. The transmission of sound through turbulent shear layers", Journal of Fluid Mechanics, 89(4), 1978, pp. 723-749.

${ }^{6}$ Campos, L., "The spectral broadening of sound by turbulent shear layers. Part 2. The spectral broadening of sound and aircraft noise", Journal of Fluid Mechanics, 89(4), 1978, pp. 751-783.

${ }^{7}$ Candel, S., Guedel, A. \& Julienne, A., "Refraction and scattering of sound in an open wind tunnel flow", 6th International Congress on Instrumentation in Aerospace Simulation Facilities, 1975, pp. 288-300.

${ }^{8}$ Candel, S., Guedel, A. \& Julienne, A., "Radiation, refraction and scattering of acoustic waves in a free shear flow", $3 r d$ AIAA Aero-Acoustics Conference, 1976.

${ }^{9}$ Colonius, T., Lele, S.K. \& Moin, P., "The scattering of sound waves by a vortex: numerical simulations and analytical solutions", Journal of Fluid Mechanics, 260, 1994, pp. 271-298.

${ }^{10}$ Ewert, R., "Broadband slat noise prediction based on CAA and stochastic sound sources from a fast Random ParticleMesh (RPM) method", Computers and Fluids, 37, 2008, pp. 369-387.

${ }^{11}$ Ewert, R., Dierke, J., Siebert, J., Neifeld, A., Appel, C., Siefert, M. \& Kornow, O., "CAA broadband noise prediction for aeroacoustic design", Journal of Sound and Vibration, 330, 2011, pp. 4139-4160.

${ }^{12}$ Ewert, R., Kornow, O., Delfs, J., Yin, J., Röber, T. \& Rose, M., "A CAA based approach to tone haystacking", 15th AIAA/CEAS Aeroacoustics Conference, n ${ }^{\circ}$ AIAA-2009-3217, 2009.

${ }^{13}$ Ewert, R., Kornow, Tester, B., Powles, C., Delfs, J. \& Rose, M., "Spectral broadening of jet engine turbine tones", 14 th AIAA/CEAS Aeroacoustics Conference, no AIAA-2008-2940, 2008.

${ }^{14}$ Ewert, R. \& Schröder, W. "On the simulation of trailing edge noise with a hybrid LES/APE method", Journal of Sound and Vibration, 270, 2004, pp. 509-524.

${ }^{15}$ Ford, R. \& Llewellyn Smith, S., "Scattering of acoustic waves by a vortex", Journal of Fluid Mechanics, 386, 1999, pp. 305-328. 
${ }^{16}$ Georges, T., "Acoustic ray paths through a model vortex with a viscous core", Journal of the Acoustical Society of America, 51, 1972, pp. 206-209.

${ }^{17}$ Goedecke, G., Wood, R., Auvermann, H., Ostashev, V., Havelock, D. \& Ting, C., "Spectral broadening of sound scattered by advecting atmospheric turbulence", Journal of the Acoustical Society of America, 109(5), 2001, pp. 1923-1934.

${ }^{18}$ Howe, M., "Multiple scattering of sound by turbulence and other inhomogeneities", Journal of Sound and Vibration, 27(4), 1973, pp. 455-476.

${ }^{19}$ Kraichnan, R., "The scattering of sound in a turbulent medium", Journal of the Acoutical Society of America, 25(6), 1953, pp. 1096-1104.

${ }^{20}$ Kröber, S., Hellmold, M. \& Koop, L., "Experimental Investigation of Spectral Broadening of Sound Waves by Wind Tunnel Shear Layers", 19th AIAA/CEAS Aeroacoustics Conference, no AIAA-2013-2255, 2013.

${ }^{21}$ Lighthill, M., "On the energy scattered from the interaction of turbulence with sound or shock waves", Mathematical Proceedings of the Cambridge Philosophical Society, 49, 1953, pp. 531-551.

${ }^{22}$ McAlpine, A., Powles, C. \& Tester, B., "A weak-scattering model for turbine-tone haystacking", Journal of Sound and Vibration, 332, 2013, pp. 3806-3831.

${ }^{23}$ Mohseni, K., \& Colonius, T., "Numerical treatment of polar coordinate singularities", Journal of Computational Physics, 157, 2000, pp. 787-795.

${ }^{24}$ O'Shea, S., "Sound scattering by a potential vortex", Journal of Sound and Vibration, 43(1), 1975, pp. 109-116.

${ }^{25}$ Powles, C., Tester, B. \& McAlpine, A., "A weak-scattering model for turbine-tone haystacking outside the cone of silence", International Journal of Aeroacoustics, 10(1), 2011, pp. 17-50.

${ }^{26}$ Sijtsma, P., Oerlemans, S., Tibbe, T., Berkefeld, T. \& Spehr, C., "Spectral broadening by shear layers of open jet wind tunnels", 20th AIAA/CEAS Aeroacoustics Conference, no AIAA-2014-3178, 2014.

${ }^{27}$ Tam, C. \& Webb, J., "Dispersion-Relation-Preserving finite difference schemes for computational acoustics", Journal of Computational Physics, 107(2), 1993, pp. 262-281.

${ }^{28}$ Thompson, K., "Time dependent boundary conditions for hyperbolic systems", Journal of Computational Physics, 68, 1987, pp. 1-24. 